\title{
Dynamic optimization and Skiba sets in economic examples
}

\author{
Wolf-Jürgen Beyn* \\ Department of Mathematics \\ email: beyn@mathematik.uni-bielefeld.de
}

\author{
Thorsten Pampel ${ }^{\dagger *}$ \\ Department of Economics \\ email: tpampel@wiwi.uni-bielefeld.de
}

\author{
Willi Semmler \\ Department of Economics \\ email: wsemmler@wiwi.uni-bielefeld.de \\ University of Bielefeld \\ P. O. Box 100131, D-33501 Bielefeld, Germany
}

Discussion Paper No. 469

\begin{abstract}
We discuss two optimization problems from economics. The first is a model of optimal investment and the second is a model of resource management. In both cases the time horizon is infinite and the optimal control variables are continuous. Typically, in these optimal control problems multiple steady states and periodic orbits occur. This leads to multiple solutions of the state-costate system each of which relates to a locally optimal strategy but has its own limiting behavior (stationary or periodic). Initial states that allow different optimal solutions with the same value of the objective function are called Skiba points. The set of Skiba points is of interest, because it provides thresholds for a global change of optimal strategies. We provide a systematic numerical method for calculating locally optimal solutions and Skiba points via boundary value problems. In parametric or higher dimensional systems Skiba curves (or manifolds) appear and we show how to follow them by a continuation process. We apply our method to the models above where Skiba sets consist of points or curves and where optimal solutions have different stationary or periodic asymptotic behavior.
\end{abstract}

Keywords: Optimal control problems, approximation method, candidates for optimal solutions, Skiba sets, multiple steady states, periodic orbits

JEL Classification: C61, C63, E22

This is a preprint of an article accepted for publication in "Optimal Control Applications and Methods" published by John Wiley \& Sons Ltd.

\footnotetext{
${ }^{*}$ Supported by DFG Schwerpunktprogramm "Ergodentheorie, Analysis und effiziente Simulation dynamischer Systeme".

†Supported by DFG under contract No. BO 635/9-1,3.
} 


\section{Introduction}

Dynamic optimization has become an important method in economics. Numerous examples have been given where dynamic optimization in economics can lead to multiple steady states and periodic orbits. In earlier growth theory it has been shown that a convex-concave production function leads to multiple steady states. Examples are given in the literature on development economics, see Skiba (1978) [32] and Azariadis \& Drazen (1990) [1]. Multiple steady states have further been studied in modern growth theory. In endogenous growth models of Lucas and Romer type multiple steady states or a continuum of steady states may arise, for the Lucas model see Benhabib, Perli \& Xie (1994) [4] and for the Romer model see Benhabib \& Perli (1994) [3] and Evans, Honkapohja \& Romer (1998) [12], see also Santos (1999) [26]. The literature on resource economics and ecological management problems show also numerous examples of models with multiple steady states (see Brock \& Starret (1999) [7] and Sieveking \& Semmler (1997) [30]). Multiple steady states solutions are also important properties in trade models (see Krugman (1991) [15]), in models of addiction (see Orphanides \& Zervos [20, 21]), in labor market search (see, for example, Mortensen (1989) [19]) and in monetary control models (see Benhabib, Schmitt-Grohe \& Uribe (1998) [5] and Semmler \& Greiner (1999) [28]).

Such systems often lead to multiple solutions of the state-costate system each of which relates to a locally optimal strategy but has its own limiting behavior (stationary or periodic). There are initial states that allow different optimal solutions with the same value for the objective function. These are called Skiba points. The set of Skiba points is of interest for economics, since it provides a threshold for a global change of optimal strategies. Our paper develops a methodology of how to numerically study such systems via the Hamiltonian and by solving boundary value problems on finite intervals. In fact, for Skiba points one of the boundary conditions states that the objective values for two trajectories are identical.

Our approach is limited to optimization problems with continuous controls. Moreover, we can at most detect finitely many locally optimal solutions with prescribed asymptotic behavior (stationary or periodic). We apply our method to two prototype dynamic models, one from modern growth theory and the other from resource economics.

Our method is based on a general technique for approximating so called generalized connecting orbits in continuous dynamical systems, as developed in [22], [24]. Setting up an appropriate boundary value problem we approximate solutions converging either to steady states or periodic orbits. The specific setup of the method used in this paper is summarized in Section 2.

In Section 3 we define the class of optimization problems with infinite horizon to which our method applies. We employ a proper version of Pontryagin's maximum principle to define the set of "candidates for optimal solutions" with "stationary or periodic asymptotic behavior". These solutions are of particular interest, because such candidates are bounded in state space and they satisfy the necessary first 
order conditions for locally optimal solutions. Connecting orbits are located in the space of state and costate variables and they determine optimal controls as well as the system response under this control, if the optimal solutions are asymptotically stationary or periodic. For the solution technique to work we assume the existence of an optimal solution (so that Pontryagin's maximum principle applies) and we restrict our analysis to solutions without jumps in order to get a continuous dynamical system.

The algorithm detects solutions with specific asymptotic behavior. Comparing the objective values (obtained by numerical integration) for different solutions we are able to discard suboptimal solutions.

The topic of 3.3 is the computation of Skiba points with stationary or periodic asymptotic behavior by using the method from [22, Chapter 5], [23].

In Section 4 we analyze a model of optimal investment and consumption policy which is presented in [29]. In particular, we approximate two solutions converging to different steady states. To the first one our method and the theory applies. This is not the case for the second solution, which converges in finite time to a singular point on the boundary of the domain. Here we derive some appropriate boundary conditions and modify our approach so that it still works in practice.

A model of optimal exploitation of renewable resources and optimal consumption policy, presented in [30], is analyzed in Section 5. Here we detect several periodic solutions and determine appropriate solutions converging to either one or the other.

\section{The numerical method}

In this section we give a survey of the numerical method and its error analysis. We are interested in solutions of a dynamical system

$$
\dot{x}=F(x), \quad x(t) \in \mathbb{R}^{m}
$$

with initial value $x_{0}$ in a given subspace $\mathcal{W} \subset \mathbb{R}^{m}$ and converging to a given steady state $(y(t) \equiv \hat{y})$ or to a $T$-periodic orbit $y(t)(y(0)=y(T))$. Therefore we approximate solutions $\xi(t)$ of

$$
\dot{\xi}(t)=F(\xi(t)), t \in \mathbb{R}_{+} \quad \text { with } \quad \xi(0) \in \mathcal{W} \quad \text { and } \quad \lim _{t \rightarrow \infty}|\xi(t)-y(t)|=0 .
$$

In the setup of this paper the subspace $\mathcal{W}$ is defined by fixing $k$ initial coordinates, i. e. $x_{\iota}(0)=\bar{x}_{\iota}$ for $\iota \in I=\left\{\iota_{1}, \ldots, \iota_{k}\right\}$ with $1 \leq \iota_{1}<\cdots<\iota_{k} \leq m$. Writing $x_{I}=\left(x_{\iota_{i}}\right)_{i=1}^{k} \in \mathbb{R}^{k}$ we can express the condition $\xi(0) \in \mathcal{W}$ as $\xi_{I}(0)=\bar{x}_{I} \in \mathbb{R}^{k}$.

We call a solution of (2) a connecting orbit from $\mathcal{W}$ to $\boldsymbol{y}$ and we call it nondegenerate if in addition the stable manifold of $y$ intersects the subspace $\mathcal{W}=\left\{x \in \mathbb{R}^{m} \mid x_{I}=\bar{x}_{I}\right\}$ transversally (for a precise definition and more general type of connecting orbits see [22]). 
In the following we assume, that there exists a nondegenerate connecting orbit which we want to approximate. Our method is based on a general technique for approximating so called generalized connecting orbits in continuous dynamical systems, as developed in [22], [24].

The main tool is a solver for boundary value problems which also allows for continuation of parameters. In the applications we use the collocation based solver COLCON/COLPAR by Bader \& Kunkel [2]. In addition one has to locate zeros of $F$ (equilibria) and to compute eigenvalues and eigenvectors in order to set up appropriate asymptotic boundary conditions.

Henceforward we assume that $F$ is sufficiently smooth (at least twice differentiable) and that $y(t)$ is a given solution of (1) which is either a hyperbolic steady state $(y(t) \equiv \hat{y})$ or a hyperbolic $T$-periodic orbit $(y(0)=y(T))$.

REMARK A steady state is called hyperbolic, if its linearization has no eigenvalue $\mu$ with $\operatorname{Re}(\mu)=0$ and a periodic orbit is called hyperbolic, if it has a simple Floquet multiplier 1 and no other Floquet multiplier on the unit circle.

\subsection{Steady states}

To detect a steady state $\hat{y}$ we solve $F(\hat{y})=0$. Then we compute the eigenvalues of $F^{\prime}(\hat{y})$ and denote the number of eigenvalues with negative real part by $m_{a}$. To obtain an orbit connecting to $\hat{y}$ one has to fix $k=m_{a}$ initial values, i. e. $\# I=m_{a}$ and $\bar{x}_{I} \in \mathbb{R}^{m_{a}}$. If $m_{a}=0$, then there is no solution converging to $\hat{y}$, except the stationary solution $\hat{y}$ itself. If $m_{a}=m$, then all solutions in a neighborhood of $\hat{y}$ converge to $\hat{y}$ and hence (2) is an initial value problem without an asymptotic condition. Therefore we assume henceforward $0<m_{a}<m$ and define $m_{b}=m-m_{a}$.

REMARK Fixing $k=m_{a}$ inital values is necessary to get a transverse intersection of the stable manifold with the subspace defined by $\left\{x \in \mathbb{R}^{m} \mid x_{I}=\bar{x}_{I}\right\}$. In the economic examples (1) is usually a state-costate system. If $m_{a}$ is larger than the number of state variables then one has to fix some costate variables (and hence there is a continuum of solutions converging to $\hat{y}$ ), but if $m_{a}$ is smaller than the number of state variables then one has to free some state variables to obtain initial values with a solution converging to $\hat{y}$.

To define an asymptotic boundary condition we compute a matrix $V \in \mathbb{R}^{m_{b}, m}$, where the rows of $V$ span the unstable subspace of $F^{\prime}(\hat{y})^{T}$, i. e. we solve

$$
V F^{\prime}(\hat{y})=U V
$$

where $U \in \mathbb{R}^{m_{b}, m_{b}}$ with $\operatorname{Re}(\sigma(U))>0$.

REMARK Here we use an eigenvalue solver which computes the eigenvectors of $F^{{ }^{T}}(\hat{y})$ and we define the rows of $V$ by the eigenvectors corresponding to the unstable eigenvalues. For a complex pair two columns are defined, one by the the real part and one by the imaginary part of the complex eigenvector. 
Using this matrix $V$ one obtains $V(x-\hat{y})=0$ if $x-\hat{y}$ is in the stable subspace of $F^{\prime}(\hat{y})$. The approximation of a solution $\bar{\xi}$ of $(2)$ on a finite interval $\left[0, T_{+}\right]$is then obtained from the solution of the boundary value problem

$$
\begin{aligned}
\dot{\xi}(t) & =F(\xi(t)), t \in\left[0, T_{+}\right], \\
\xi_{\iota}(0) & =\bar{x}_{\iota}, \iota \in I \\
V\left(\xi\left(T_{+}\right)-\hat{y}\right) & =0
\end{aligned}
$$

REMARK A main problem is to define a "good" initial approximation of the boundary value problem (4.a-4.c). One strategy is, to start in the stable subspace of $\hat{y}$, integrate backwards and choose as a final value some $\tilde{x}_{I}$ which comes close to $\mathcal{W}$. This defines a solution of (4.a), (4.c) with initial value $\tilde{x}_{I}$. The coordinates of $\xi_{I}(0)$ are then successively used as continuation parameters in (4.a-4.c) until $\xi_{I}(0)=\bar{x}_{I}$ is satisfied. A similar strategy, called "successive continuation", is developed in [10].

If $F$ depends on parameters and one wants to get solutions for different parameter values, it is useful to compute the steady state, the asymptotic boundary condition and the solution from a single parameter dependent system as follows:

$$
\left(\begin{array}{c}
F(\hat{y}, \lambda) \\
\dot{\xi}-F(\xi, \lambda) \\
\xi_{I}(0)-\bar{x}_{I} \\
V\left(\xi\left(T_{+}\right)-\hat{y}\right) \\
V \frac{\partial}{\partial x} F(\hat{y}, \lambda)-U V \\
V V^{T}-\hat{V} \hat{V}^{T}
\end{array}\right)=0 .
$$

Here $\lambda$ is the continuation parameter and the unknowns are the steady state $\hat{y}$, the matrices $V$ and $U$, and the asymptotic solution $\xi$. In addition $\hat{V} \in \mathbb{R}^{m_{b}, m}$ is a normalizing matrix obtained from the eigenvalue problem (3) at an initial parameter $\hat{\lambda}$.

\subsection{Periodic solutions}

To detect a $\bar{T}$-periodic solution $y$ we solve the $(n+1)$-dimensional boundary value problem

$$
\begin{aligned}
\dot{\eta}(t) & =T F(\eta(t)), \dot{T}(t)=0, t \in[0,1], \quad \eta(0)=\eta(1) \\
\tilde{\chi}(\eta) & =0
\end{aligned}
$$

where $\tilde{\chi}$ is a scalar phase condition, e. g. $\tilde{\chi}(\eta)=F(\tilde{\eta})^{T}(\eta(0)-\tilde{\eta})$ with $\tilde{\eta}$ in a neighborhood of the (expected, but yet undetected) period orbit. The result is the period $\bar{T}$ and a 1-periodic solution $\eta_{0}$ of the scaled system (6), hence $y(t):=\eta_{0}(t / \bar{T})$ is a $\bar{T}$-periodic solution of (1). Systems of the type (6), (7) are due to [9], [14].

In addition to $\eta_{0}$, each "phase shifted solution" solves (6). In the later applications we usually cannot fix the phase of the periodic orbit as in (7). Rather we 
introduce an additional phase parameter $\varphi$ and the value of $\varphi$ becomes part of the problem (for determining optimal solutions). To specify the periodic orbit which corresponds to a phase parameter $\varphi$ we define a phase fixing condition $\chi(\eta, \varphi)=0$ which depends smoothly on $\varphi$ and satisfies $\chi\left(\eta_{0}, 0\right)=0$ (examples for $\chi$ are given below). We replace (6), (7) by

$$
\begin{aligned}
\dot{\eta}(t) & =T F(\eta(t)), t \in[0,1], \\
\dot{T}(t) & =0, \\
\dot{\varphi}(t) & =0 \\
\eta(0) & =\eta(1), \\
\chi(\eta, \varphi) & =0 .
\end{aligned}
$$

Its solution $\left(\eta_{\varphi}, \bar{T}\right)$ is the periodic solution corresponding to $\varphi$.

REMARKS 1. The function $\chi$ should be chosen in such a way that the existence of a phase shift (and hence a periodic solution) satisfying the phase condition can be guaranteed. An example for a phase fixing function is $\chi(\eta, \varphi)=$ $\dot{\eta}_{0}(\varphi)^{T}\left(\eta(0)-\eta_{0}(\varphi)\right)$, where $\eta_{0}$ is a periodic solution already calculated and the additional parameter $\varphi$ is the phase shift itself. If we expect $\varphi$ to be small, then instead of computing $\eta_{0}$ we can use for $\chi(\eta, \varphi)$ a simpler expression based on the initial approximation $\tilde{\eta}$ like $\chi(\eta, \varphi)=F(\tilde{\eta})^{T}(\eta(0)-(\tilde{\eta}+\varphi F(\tilde{\eta}))$. In the application in Section 5 we choose yet another phase condition described there.

2. The problem of detecting periodic orbits and hence a sufficiently good initial approximation for the boundary value problem remains. In the application in Section 5 we use the continuation software CONTENT [17] for this task.

Let $U$ be the solution of the variational equation $\dot{U}=\bar{T} F^{\prime}\left(\eta_{\varphi}(\cdot)\right) U, U(0)=\operatorname{Id}_{\mathbb{R}^{\mathrm{m}, \mathrm{m}}}$. Then the eigenvalues $\mu$ of $U(1)$ are the Floquet multipliers and we denote the number of stable Floquet multipliers (i. e. $|\mu|<1$ ) by $m_{a}$. Since 1 is a Floquet multiplier we have $m_{b}:=m-m_{a}>0$. As in the steady state case we fix the number $k$ of given initial values by the dimension of the stable manifold of the periodic orbit and hence $\# I=k=m_{a}+1$ (the center direction corresponding to the Floquet multiplier 1 is part of the stable manifold).

To define appropriate asymptotic boundary conditions we solve the adjoint variational equation

$$
\dot{W}=-W \bar{T} F^{\prime}\left(\eta_{\varphi}(\cdot)\right) \quad \text { with } \quad W(0)=\operatorname{Id}_{\mathbb{R}^{\mathrm{m}, \mathrm{m}}} .
$$

From the fundamental matrix $W$ we need only a submatrix $V_{\varphi}$ the rows of which belong to the eigenvalues $|\mu| \leq 1$ of $W(1)$. For this purpose we solve

$$
\dot{V}=-V \bar{T} F^{\prime}\left(\eta_{\varphi}(\cdot)\right) \quad \text { with } \quad V(1)=\Lambda V(0),
$$

for $V(t) \in \mathbb{R}^{m_{b}, m}$ and some $\Lambda \in \mathbb{R}^{m_{b}, m_{b}}$ satisfying $|\mu| \leq 1$ for all eigenvalues $\mu$ of $\Lambda$. Then we set $V_{\varphi}=V(0)$. 
If we assume that we can compute $\eta_{\varphi}$ and $V_{\varphi}$ smoothly in $\varphi$, then we approximate the solution $(\bar{\xi}, \bar{\varphi})$ of the scaled problem

$$
\dot{\xi}(t)=\bar{T} F(\xi(t)), t \in \mathbb{R}_{+} \quad \text { with } \quad \xi_{I}(0)=\bar{x}_{I} \quad \text { and } \quad \lim _{t \rightarrow \infty}\left|\xi(t)-\eta_{\varphi}(t)\right|=0
$$

on a finite interval $J=\left[0, T_{+}\right], T_{+} \in \mathbb{N}$ by the solution $\left(\xi_{J}, \varphi_{J}\right)$ of the boundary value problem

$$
\begin{aligned}
\dot{\xi}(t) & =\bar{T} F(\xi(t)), t \in\left[0, T_{+}\right], \\
\xi_{I}(0) & =\bar{x}_{I}, \\
V_{\varphi}\left(\xi\left(T_{+}\right)-\eta_{\varphi}\left(T_{+}\right)\right) & =0 .
\end{aligned}
$$

Note that the approximation $\xi_{J}$ has been scaled, so that $\xi_{J}(t / \bar{T}), 0 \leq t \leq T_{+} \bar{T}$ approximates the solution of the original system $(2)$ on $\left[0, T_{+} \bar{T}\right]$.

REMARK Above we have assumed $T_{+} \in \mathbb{N}$ for simplicity. For more general $T_{+} \in$ $\mathbb{R}_{+}$we can keep (11) but need to replace (9) by

$$
\dot{V}=-V \bar{T} F^{\prime}\left(\eta_{\varphi}\left(\cdot+T_{+}\right)\right) \text {with } \quad V(1)=\Lambda V(0) .
$$

Notice that in case $T_{+} \in \mathbb{N}$ both equations agree.

Typically, computing $\eta_{\varphi}$ and $V_{\varphi}$ smoothly in $\varphi$ is cumbersome because during the solution procedure for (11) the large system (9) has to be solved many times. Therefore we use a simpler approach, called the boundary corrector method.

1. Select an initial approximation $\varphi_{0}$.

2. Compute $V_{\varphi_{0}}$ by solving (9) for $\varphi_{0}$.

3. Calculate $\left(\xi_{J}, \varphi_{J}, \eta_{\varphi_{J}}, \bar{T}\right)$, the solution of

$$
G_{J}^{p}(\xi, \varphi, \eta, T):=\left(\begin{array}{c}
\dot{\eta}-T F(\eta) \\
\chi(\eta, \varphi) \\
\eta(0)-\eta(1) \\
\dot{\xi}-T F(\xi) \\
\xi_{I}(0)-\bar{x}_{I} \\
V_{\varphi_{0}}\left(\xi\left(T_{+}\right)-\eta\left(T_{+}\right)\right)
\end{array}\right)=0 .
$$

4. Repeat step 1. with $\varphi_{0}=\varphi_{J}$ and step 2. and terminate.

REMARK The first three lines of (12) define a periodic orbit $\eta$ and the period $\bar{T}$ corresponding to the phase $\varphi$, the fourth line defines the dynamical system, the fifth line fixes the $m_{a}+1$ initial values and the sixth line defines the asymptotic boundary conditions.

\subsection{Approximation theorem}

The numerical method is supported by an error analysis which was derived for a more general setting in [22], [24]. Here we restrict the method to the cases of steady states (Theorem 1) and periodic orbits (Theorem 2). 
THEOREM 1 Suppose that $\tilde{x}$ is a nondegenerate connecting orbit from $\left\{x \in \mathbb{R}^{m} \mid x_{I}=\right.$ $\left.\bar{x}_{I}\right\}$ to a steady state $\hat{y}$ and let $\gamma<0, \beta \geq 0$ satisfy $\operatorname{Re}(\mu)<\gamma$ for all eigenvalues of $F^{\prime}(\hat{y})$ with negative real part and $\operatorname{Re}(\mu)>\beta$ for all eigenvalues of $F^{\prime}(\hat{y})$ with positive real part.

Then there exist $\delta>0, C>0$ and $\bar{T}_{+}>0$ sufficiently large, such that for $T_{+} \geq \bar{T}_{+}$ there exists a neighborhood $\mathcal{B}\left(\delta, \beta, T_{+}\right)$of $\left.\tilde{x}\right|_{J}$ (restricted to $J=\left[0, T_{+}\right]$) in which the operator equation (5) (or (4.a-4.c) respectively) has a unique solution $\xi_{J}$.

Furthermore, the following estimate holds

$$
\left|\xi_{J}(t)-\tilde{x}(t)\right| \leq C e^{(2 \gamma-\beta) T_{+}}, 0 \leq t \leq T_{+} .
$$

In the case of periodic orbits we state the theorem according to the boundary corrector method.

THEOREM 2 Suppose that $\tilde{x}$ is a nondegenerate connecting orbit from $\left\{x \in \mathbb{R}^{m} \mid x_{I}=\right.$ $\left.\bar{x}_{I}\right\}$ to $\eta_{\tilde{\varphi}}$ in the scaled system (10) and let $\gamma<0$ satisfy $|\mu|<e^{\gamma}$ for all Floquet multipliers within the unit circle.

Then there exist $\delta>0, C>0$ and $\bar{T}_{+}>0$ sufficiently large, such that for $T_{+} \geq \bar{T}_{+}$ there exists a neighborhood $\mathcal{B}\left(\delta, T_{+}\right)$of $\left.\tilde{x}\right|_{J}$ (restricted to $J=\left[0, T_{+}\right]$) such that the boundary corrector method defined by (12) has a unique solution $\left(\xi_{J}, \varphi_{J}\right)$ in $\mathcal{B}\left(\delta, T_{+}\right)$.

Furthermore, the following estimates hold

$$
\begin{aligned}
\left|\varphi_{J}-\tilde{\varphi}\right| & \leq C e^{2 \gamma T_{+}} \\
\left|\xi_{J}(t)-\tilde{x}\right|_{J}(t) \mid & \leq C e^{2 \gamma T_{+}}, 0 \leq t \leq T_{+} .
\end{aligned}
$$

REMARK The theoretical results are derived for $F$ defined on all of $\mathbb{R}^{m}$. Nevertheless, both, the numerical method and the error estimates work in a neighborhood of the exact solution. Hence we can apply the results to $F$, defined on a subset $U \subset \mathbb{R}^{m}$, provided that the exact solution and the steady state or periodic orbit are in the interior of $U$.

\section{The general setup}

In this section we follow [18] and define a class of optimization problems with infinite horizon to which the methods of Section 2 apply. Using Pontryagin's maximum principle we arrive at a continuous dynamical system as in (1) if we assume continuity of the control variables. We apply the techniques from Section 2 to detect continuous solutions which satisfy the necessary first order conditions (hence they have a chance to be optimal and will be called candidates in 3.2) and which have stationary or periodic asymptotic behavior. 
The methods of Section 2 require a twice differentiable function and therefore, we cannot deal with systems where solutions with jumps in the control are optimal. Moreover, it is obvious that an optimal solution can only be detected by this method, if it has the prescribed asymptotic behavior. Yet the method allows to rule out suboptimal solutions.

The optimization problem is to maximize the objective function

$$
\hat{U}(x, c):=\int_{0}^{\infty} e^{-\theta t} U(x(t), c(t)) \mathrm{dt}
$$

subject to

$$
\dot{x}(t)=f(x(t), c(t)), t \in \mathbb{R}_{+}, \quad x(0)=x_{0},
$$

where $x(t) \in \mathbb{R}^{n}$ are the state variables and $c(t) \in \mathbb{R}^{n}$ are the control variables which are assumed to be piecewise continuous. Moreover, we assume $U \in C^{3}\left(\mathbb{R}^{2 n}, \mathbb{R}\right)$, $f \in C^{3}\left(\mathbb{R}^{2 n}, \mathbb{R}^{n}\right)$.

Definition 3 A pair $(x(\cdot), c(\cdot))$ is called an admissible trajectory, if $x(\cdot)$ is a solution of (14) with control $c(\cdot)$, except at the jumps of $c$, and if the integral (13) converges. The set of all admissible trajectories is denoted by $\mathcal{T}_{a d}\left(x_{0}\right)$.

A pair $(\bar{x}(\cdot), \bar{c}(\cdot))$ is called an optimal solution of the problem (13), (14), if it is admissible and if it maximizes (13) over all admissible pairs $(x(\cdot), c(\cdot)) \in \mathcal{T}_{\text {ad }}\left(x_{0}\right)$. The set of all optimal solutions is denoted by $\mathcal{T}_{\text {opt }}\left(x_{0}\right)$.

A first observation implied by recurrence in the state variables is stated in the following proposition.

Propositon 4 If there is an optimal solution $(x(\cdot), c(\cdot))$ with $x(0)=x(T), T>0$, then the following solutions are optimal:

A: The solution itself, i. e. $\left(x_{A}(\cdot), c_{A}(\cdot)\right)=(x(\cdot), c(\cdot))$,

$B$ : The solution starting at $x(T)$ with control $c(\cdot+T)$, i. e.

$$
\left(x_{B}(\cdot), c_{B}(\cdot)\right)=(x(\cdot+T), c(\cdot+T))
$$

C: The T-periodic solution which switches at each $n T, n \in \mathbb{N}$ to $x(0)$, i. e.

$$
\left(x_{C}(\cdot), c_{C}(\cdot)\right)=(x(\cdot \bmod T), c(\cdot \bmod T)) .
$$

Proof Breaking up the integral in (13) and using the geometric series one obtains

$$
\begin{aligned}
\hat{U}\left(x_{B}, c_{B}\right) & =\int_{0}^{\infty} e^{-\theta t} U(x(t+T), c(t+T)) \mathrm{dt}=\frac{1}{e^{-\theta T}} \int_{T}^{\infty} e^{-\theta t} U(x(t), c(t)) \mathrm{dt} \\
\hat{U}\left(x_{C}, c_{C}\right) & =\sum_{i=0}^{\infty} \int_{0}^{T} e^{-\theta(t+i T)} U(x(t+i T), c(t+i T)) \mathrm{dt} \\
& =\frac{1}{1-e^{-\theta T}} \int_{0}^{T} e^{-\theta t} U(x(t), c(t)) \mathrm{dt}
\end{aligned}
$$


and

$$
\hat{U}\left(x_{A}, c_{A}\right)=\int_{0}^{\infty} e^{-\theta t} U(x(t), c(t)) \mathrm{dt}=e^{-\theta T} \hat{U}\left(x_{B}, c_{B}\right)+\left(1-e^{-\theta T}\right) \hat{U}\left(x_{C}, c_{C}\right) .
$$

Hence Proposition 4 follows from the optimality of solution A.

REMARK If $(x(\cdot), c(\cdot))$ is T-periodic, then the solutions $A, B$ and $C$ coincide.

It is not our intention to prove existence of optimal solutions, hence we assume:

Assumption 1: There exists at least one optimal solution of the problem (13),

REMARK For sufficient conditions for optimality in infinite horizon problems see e. g. [27]. However, in our applications the Hamiltonian is not concave in the state variables and hence the theorems of that paper do not apply.

The technique used here is to apply Pontryagin's maximum principle to detect candidates for optimal solutions. These are admissible trajectories which satisfy the necessary conditions for optimal solutions stated in the forthcoming Theorem 5. For these solutions we compute the values of the objective function (13) by numerical integration and choose those with maximum value. We do not claim that these solutions are optimal, but we can state that the remaining ones are suboptimal.

The following theorem is a special version of Pontryagin's maximum principle from $[18$, Theorem A].

TheOREM 5 A necessary condition for an admissible trajectory $(\bar{x}(\cdot), \bar{c}(\cdot)) \in \mathcal{T}_{\text {ad }}\left(x_{0}\right)$ to be an optimal solution of (13), (14) is, that there exist $\lambda \in\{0,1\}$ and an initial costate value $y_{0} \in \mathbb{R}^{n}$, such that

1. $\left(\lambda, y_{0}\right) \neq 0$.

2. The costate variable $y(\cdot)$ is a solution of the initial value problem

$$
\begin{aligned}
& \dot{y}(t)=-\lambda e^{-\theta t}\left(\frac{\partial}{\partial x} U(\bar{x}(t), \bar{c}(t))\right)^{T}-\left(\frac{\partial}{\partial x} f(\bar{x}(t), \bar{c}(t))\right)^{T} \cdot y(t), \\
& y(0)=y_{0} .
\end{aligned}
$$

3. For each $t \in \mathbb{R}_{+}$where $\bar{c}(t)$ is continuous, $(\bar{x}(t), \bar{c}(t), y(t), \lambda)$ satisfies

$$
H(\bar{x}(t), \bar{c}(t), y(t), \lambda, t)=\max _{c} H(\bar{x}(t), c, y(t), \lambda, t),
$$

where $H$ is the Hamiltonian function

$$
H(x, c, y, \lambda, t):=\lambda e^{-\theta t} U(x, c)+f(x, c)^{T} \cdot y .
$$


4. The maximum value of the Hamiltonian satisfies for every $t$

$$
M(t):=H(\bar{x}(t), \bar{c}(t), y(t), \lambda, t)=-q(t),
$$

where the function $q(t) \in \mathbb{R}$ is the solution of

$$
\begin{aligned}
& \dot{q}=\lambda \theta e^{-\theta t} U(\bar{x}(t), \bar{c}(t)), \\
& \lim _{t \rightarrow \infty} q(t)=0 .
\end{aligned}
$$

Proof See [18, Theorem A].

REMARK The initial costate value $y_{0}$ is unknown. If $y_{0}$ and $\bar{c}(\cdot)$ were given, then (14) and (15) constitute an initial value problem for $x$ and $y$.

For admissible $(\bar{x}, \bar{c})$ the integral (13) converges (see Definition 3) and hence (18) leads for $\lambda=1$ to the formula

$$
q(t)=-\theta \int_{t}^{\infty} e^{-\theta s} U(\bar{x}(s), \bar{c}(s)) \mathrm{ds} .
$$

In this section we will henceforward assume $\lambda=1$. In the applications such a "normality condition" requires a proof (see e. g. [33]).

In particular, we deal with systems where we can express $\bar{c}(t)$ by $(16)$ in terms of $\bar{x}(t)$ and the scaled costate function $z(t):=e^{\theta t} y(t)$, i. e. we can write $\bar{c}(t)=$ $C(\bar{x}(t), z(t))$. Thus we assume:

Assumption 2: Any candidate for an optimal solution satisfies $\lambda=1$ and there exists a twice differentiable function $C$, such that $C(x, z)$ maximizes the scaled Hamiltonian function $\tilde{H}(x, c, z):=U(x, c)+f(x, c)^{T} \cdot z$ for all $(x, z) \in \mathbb{R}^{2 n}$.

REMARK If the Hamiltonian is concave in the control variables, then the equation $\frac{\partial}{\partial c} \tilde{H}(x, c, z)=0$ defines for each $(x, z)$ the maximizing $C(x, z)$ except at the boundary of the control set. The function $C$ is twice differentiable if the Hamiltonian is sufficiently smooth and if $C(x, z)$ is in the interior of the control set.

Both, the state and the costate variables are continuous for an optimal solution and hence the control is continuous by assumption 2. Thus we arrive at:

Propositon 6 Assumption 2 implies that the control is continuous.

Using $z(t)=e^{\theta t} y(t), \bar{c}(t)=C(\bar{x}(t), z(t))$, the equations (14) and (15) imply that $(\bar{x}(t), z(t))$ solves the autonomous dynamical system

$$
\left(\begin{array}{c}
\dot{x} \\
\dot{z}
\end{array}\right)=\left(\begin{array}{c}
f(x, C(x, z)) \\
\theta z-\left(\frac{\partial}{\partial x} U(x, C(x, z))\right)^{T}-\left(\frac{\partial}{\partial x} f(x, C(x, z))\right)^{T} z
\end{array}\right)=: F(x, z)
$$

with $(x(0), z(0))=\left(x_{0}, y_{0}\right)$, if $(\bar{x}(\cdot), \bar{c}(\cdot))$ is an optimal solution.

We consider (20) as initial value problem depending on $y_{0} \in \mathbb{R}^{n}$ and denote its solution by $x\left(y_{0}, t\right), z\left(y_{0}, t\right)$. We abbreviate the solution functions by $x\left(y_{0}\right):=$ $x\left(y_{0}, \cdot\right), z\left(y_{0}\right):=z\left(y_{0}, \cdot\right)$ and the corresponding control by $c\left(y_{0}\right)=c\left(y_{0}, \cdot\right):=$ $C\left(x\left(y_{0}, \cdot\right), z\left(y_{0}, \cdot\right)\right)$. 


\subsection{Candidates for optimal solutions}

DEFINITION 7 An admissible trajectory $(x(\cdot), c(\cdot)) \in \mathcal{T}_{\text {ad }}\left(x_{0}\right)$ is called a candidate for an optimal solution or for short a candidate, if it satisfies the necessary conditions of Theorem 5 . The value $y_{0}$ is called the corresponding costate value and the set of all candidates for optimal solutions is denoted by $\mathcal{T}_{\text {can }}\left(x_{0}\right)$.

REMARK In the literature a solution which satisfies the necessary conditions 1.3. is often called an equilibrium (here a candidate if 4. is also satisfied) and a stationary solution is called a steady state-equilibrium (here simply a steady state), e. $g .[5]$.

Suppose that the assumptions 1 and 2 hold, then we reformulate the optimization problem and maximize

$$
\bar{U}\left(y_{0}\right):=\int_{0}^{\infty} e^{-\theta t} U\left(x\left(y_{0}, t\right), c\left(y_{0}, t\right)\right) \mathrm{dt}
$$

over all $y_{0} \in \mathbb{R}^{n}$, for which $\left(x\left(y_{0}\right), c\left(y_{0}\right)\right)$ is a candidate. The conditions 1.-3. of Theorem 5 are satisfied for all $\left(x\left(y_{0}\right), c\left(y_{0}\right)\right)$. Thus, for the set of candidates we include the conditions that the integral (21) exists and that the optimality (17) holds and get

$\mathcal{T}_{\text {can }}\left(x_{0}\right)=\left\{\left(x\left(y_{0}\right), c\left(y_{0}\right)\right) \mid y_{0} \in \mathbb{R}^{n}\right.$ and the integral (21) exists and (17) holds $\}$.

Propositon 8 Suppose that the assumptions 1 and 2 hold and that $(x, c)$ is a candidate with initial costate variable $y_{0}$ and with $x(T)=x_{0}$ and $c(T) \neq c(0)$ for some $T>0$. Then $(x, c)$ cannot be optimal.

Otherwise the $T$-periodic solution $(x(\cdot \bmod T), c(\cdot \bmod T))$ which jumps at $T$ to $\left(x_{0}, y_{0}\right)$ is also optimal (trajectory $\mathrm{C}$ in Proposition 4), but its control is not continuous at $T$. This contradicts Proposition 6.

Additional information whether a solution is optimal can be obtained from (17) and (19). If $(\bar{x}, \bar{c})$ is a candidate, then we have

$$
\int_{t}^{\infty} e^{-\theta s} U(\bar{x}(s), \bar{c}(s)) \mathrm{d} s=\frac{e^{-\theta t}}{\theta}\left(U(\bar{x}(t), \bar{c}(t))+f(\bar{x}(t), \bar{c}(t))^{T} z(t)\right) .
$$

Maximizing this at $t=0$, i. e.

$$
v\left(x_{0}, y_{0}\right)=\frac{1}{\theta}\left[U\left(x_{0}, C\left(x_{0}, y_{0}\right)\right)+f\left(x_{0}, C\left(x_{0}, y_{0}\right)\right)^{T} y_{0}\right]
$$

over all initial values of costate variables $y_{0} \in \mathbb{R}^{n}$ does not solve the original problem, because $y_{0}$ has to be restricted to initial values of costates which correspond to candidates. However, we can test (22) at $t=0$ for single admissible trajectories by numerical integration to see whether they satisfy (17) or not. Moreover, (22) 
is of particular importance for our approximation method, because it allows to determine the value of the objective function in terms of the initial values of an optimal solution.

REMARK The equation (22) always holds true for steady states $(\bar{x}, z)$ of (20) and $\bar{c}=C(\bar{x}, z)$. If $(\bar{x}(t), z(t))$ is an optimal T-periodic solution, then one can show (using periodicity and the geometric series) that (22) is equivalent to

$$
\int_{0}^{T} e^{-\theta s} U(\bar{x}(s+t), \bar{c}(s+t)) \mathrm{d} \mathrm{s}=\frac{1-e^{-\theta T}}{\theta}\left(U(\bar{x}(t), \bar{c}(t))+f(\bar{x}(t), \bar{c}(t))^{T} z(t)\right)
$$

for all $t \in[0, T]$. Hence, testing (22) for periodic orbits requires only integration on a finite interval.

\subsection{Asymptotic solutions}

Using the techniques of [22, Chapter 3] we approximate candidates for optimal solutions with special asymptotic behavior, i. e. those solutions which converge to given solutions $\eta$. In particular, we restrict our study to the case where $\eta$ is either a steady state or a periodic solution.

We consider a finite set $\mathcal{Y}=\left\{\eta_{i}\right\}_{i \in I}, I=\{1, \ldots, l\}$ of stationary or periodic solutions of $(20)$, where $\eta_{i}(t) \in \mathbb{R}^{m}, m=2 n$. In the applications the $\eta_{i}$ are either given or computing them is part of the problem. Our purpose is to decide for given $x_{0} \in \mathbb{R}^{n}$ whether there are solutions converging to any of the $\eta_{i}$ and to rule out among these the suboptimal solutions. Thus we define

Definition 9 Suppose that the assumptions 1 and 2 hold.

$A$ candidate for an optimal solution $(x(\cdot), c(\cdot)) \in \mathcal{T}_{\text {can }}\left(x_{0}\right)$ with corresponding costate value $y_{0}$ is called asymptotic to $\mathcal{Y}$, if the solution $\xi:=\left(x, z\left(y_{0}\right)\right)$ of (20) is asymptotic to some $\eta_{i} \in \mathcal{Y}$, i. e. $\lim _{t \rightarrow \infty}\left\|\xi(t)-\eta_{i}(t)\right\|=0$. The set of these solutions is denoted by $\mathcal{T}_{\text {asy }}(\mathcal{Y})\left(x_{0}\right)$.

We call a candidate $(\bar{x}(\cdot), \bar{c}(\cdot)) \in \mathcal{T}_{\text {asy }}(\mathcal{Y})\left(x_{0}\right)$ optimal with respect to $\mathcal{Y}$, if it maximizes the objective function over all $(x, c) \in \mathcal{T}_{\text {asy }}(\mathcal{Y})\left(x_{0}\right)$, i. e.

$\hat{U}(\bar{x}(\cdot), \bar{c}(\cdot))=\max _{(x, c) \in \mathcal{T}_{a s y}(\mathcal{Y})\left(x_{0}\right)}(\hat{U}(x, c))$, where $\hat{U}(x, c)=\int_{0}^{\infty} e^{-\theta t} U(x(t), c(t)) \mathrm{dt}$.

The set of candidates which are optimal with respect to $\mathcal{Y}$ is denoted by $\mathcal{T}_{a s y}^{o p t}(\mathcal{Y})\left(x_{0}\right)$.

Notice the obvious relation

$$
\mathcal{T}_{\text {asy }}^{\text {opt }}(\mathcal{Y})\left(x_{0}\right) \subset \mathcal{T}_{\text {asy }}(\mathcal{Y})\left(x_{0}\right) \subset \mathcal{T}_{\text {can }}\left(x_{0}\right) \subset \mathcal{T}_{a d}\left(x_{0}\right) .
$$

We restrict to $\eta_{i}$ which are hyperbolic, i. e. the linearization of a steady state has no eigenvalue $\mu$ with $\operatorname{Re}(\mu)=0$ or a periodic orbit has a simple Floquet multiplier 1 and no other multiplier on the unit circle, respectively. 
Moreover, we define $q_{i} \in\{-n, \ldots, n\}$, such that $n+q_{i}$ is the dimension of the stable manifold of $\eta_{i}$, i. e. in the stationary case $n+q_{i}=m_{a}$ and in the periodic case $n+q_{i}=m_{a}+1$. Let $\bar{x}_{0} \in \mathbb{R}^{n}$ be a given initial value of (14) and let $\mathcal{Y}$ be the set of solutions to which an optimal solution may converge.

\section{Analyzing asymptotic candidates}

1. Compute the $\eta_{i}$ by solving $F(\eta)=0$ or (6), (7), respectively.

2. Compute $m_{a}$, the number of stable eigenvalues (and $q_{i}=m_{a}-n$ ) or stable Floquet multiplier (and $q_{i}=m_{a}+1-n$ ), respectively, and define $m_{b}=m-m_{a}$.

3. Compute asymptotic boundary conditions $V \in \mathbb{R}^{m_{b}, m}$ by solving (3) or (9), respectively.

4. Try to find solutions converging to $\eta_{i}$.

(a) If $q_{i}=0$, then we fix the $n$ initial state values $\bar{x}_{0}$ and apply either (5) or the boundary corrector method (12).

(b) If $q_{i}<0$, i. e. $\eta_{i}$ has a stable manifold of dimension less than $n$, then generically it will not intersect in the state-costate space $\mathbb{R}^{2 n}$ the $n$ dimensional affine subspace $\left\{\bar{x}_{0}\right\} \times \mathbb{R}^{n}$ of initial values of (20). In this case we fix only $n+q_{i}<n$ inital state values and apply either (5) or the boundary corrector method (12). A solution of this procedure defines an initial state value where a solution converging to $\eta_{i}$ is possible, but in general this does not coincide with $\bar{x}_{0}$.

(c) If $q_{i}>0$, then there exists typically a $q_{i}$-dimensional surface of candidates which are asymptotic to $\eta_{i}$. This leads to the problem of maximizing $\bar{U}\left(y_{0}\right)((21))$ on a $q_{i}$-dimensional surface. To approximate a solution on this surface we fix the initial state values $\bar{x}_{0}$ and additional $q_{i}$ costate variables and apply either (5) or the boundary corrector method (12). To get further solutions on this surface we use the fixed costate variables for parameter continuation.

Let $\left(x_{i}, z_{i}\right)$ be the corresponding solution and define $c_{i}=C\left(x_{i}, z_{i}\right)$.

5. Test (22) by numerical integration to decide whether these solutions are candidates or not.

6. Choose those solutions which maximize the objective function (13) and hence rule out suboptimal ones.

REMARKS 1. We need sufficiently good initial guesses for the solutions of (5) or (12), respectively. Here we use the strategy described in Section 2 as successive continuation.

2. The method in 4.(c) only computes single solutions on the surface and it is not clear how to find an optimum on this surface. One strategy might be successive continuation of fixed costate variables in the direction where the objective function grows until it reaches a local maximum. For an analysis and implementation of such methods see [11]. 


\subsection{Skiba points}

Now we define "Skiba" points [32] and derive an approximation procedure based on the results of [22, Chapter 5], [23].

Definition 10 Suppose that assumption 1 holds. We call an initial value $x_{0} \in$ $\mathbb{R}^{n}$ a Skiba point, if there are multiple optimal solutions. Suppose in addition assumptions 2, then we call an initial value $x_{0} \in \mathbb{R}^{n}$ a Skiba point with respect to $\mathcal{Y}$, if there are multiple candidates which are optimal with respect to $\mathcal{Y}$. The set of all Skiba points with respect to $\mathcal{Y}$ is called Skiba set with respect to $\mathcal{Y}$ and it is denoted by $\mathcal{S}(\mathcal{Y})$.

In this section we restrict to hyperbolic $\eta \in \mathcal{Y}$ with $n$-dimensional stable manifolds.

In order to approximate Skiba points with respect to $\mathcal{Y}=\left\{\eta_{+}, \eta_{-}\right\}$(two alternatives) we have to solve two problems of the type in 3.2 and detect initial state values where the values of the objective functions are equal.

At first we approximate simultaneously two solutions $\left(x_{ \pm}(t), z_{ \pm}(t)\right) \in \mathbb{R}^{2 n}$ which converge to different $\eta_{+}, \eta_{-} \in \mathcal{Y}$ and have the same initial state value $x_{0}$. The corresponding controls are denoted by $c_{ \pm}(t)=C\left(x_{ \pm}(t), z_{ \pm}(t)\right)$. This is done in an obvious way by doubling the system and possibly scaling the differential equations.

To detect a Skiba point we define one coordinate $j$ of the initial state value $x_{0} \in \mathbb{R}^{n}$ as unknown parameter. Thus we substitute the conditions $x_{+_{j}}(0)=x_{0_{j}}, x_{-_{j}}(0)=$ $x_{0_{j}}$ by $x_{+_{j}}(0)=x_{-_{j}}(0)$ and the condition that the values of the objective functions are the same. We abbreviate for $x \in \mathbb{R}^{l}, l \geq n$ the vector of the first $n$ coordinates except the $j$-th by $x^{*}=\left(x_{i}\right)_{\substack{i=1, \ldots, n \\ i \neq j}} \in \mathbb{R}^{n-1}$. To define the second new condition we employ (23) for both solutions to achieve a condition which only depends on the initial values. Hence the conditions at $t=0$ are $x_{+}^{*}(0)=x_{0}^{*}, x_{-}^{*}(0)=x_{0}^{*}$, $x_{+_{j}}(0)=x_{-_{j}}(0)$ and $v\left(x_{+}(0), z_{+}(0)\right)=v\left(x_{-}(0), z_{-}(0)\right)$ with $v$ given by $(23)$.

To detect a Skiba point, where $\eta_{-}$and $\eta_{+}$are steady states we assume that $V_{-}$ and $V_{+}$are computed by (3) and we solve the boundary value problem

$$
G_{J}^{S k}\left(\xi_{-}, \xi_{+}\right):=\left(\begin{array}{c}
\dot{\xi}_{-}-\tau_{+} F\left(\xi_{-}\right) \\
\dot{\xi}_{+}-\tau_{-} F\left(\xi_{+}\right) \\
\xi_{-}^{*}(0)-x_{0}^{*} \\
\xi_{+}^{*}(0)-x_{0}^{*} \\
\xi_{+_{j}}(0)-\xi_{-j}(0) \\
v\left(\xi_{+}(0)\right)-v\left(\xi_{-}(0)\right) \\
V_{-}\left(\xi_{-}\left(T_{+}\right)-\eta_{-}\right) \\
V_{+}\left(\xi_{+}\left(T_{+}\right)-\eta_{+}\right)
\end{array}\right)=0
$$

Here the $\tau_{ \pm}$are scaling factors which can be chosen appropriately. This allows us to solve both parts $( \pm)$ of the boundary value problem on the same finite interval $\left[0, T_{+}\right]$, while the un-scaled solutions are approximated on different intervals $\left[0, \tau_{+} T_{+}\right]$and $\left[0, \tau_{-} T_{+}\right]$. 
To approximate a higher dimensional Skiba set, we can use the initial state variables (except the $x_{0_{j}}$ above) as continuation parameters.

CONCLUSION 11 If $\bar{x}_{0}$ is a Skiba point and there is a neighborhood $V$ of $\bar{x}_{0}$, such that for each $x_{0} \in V$ there exist solutions $\left(x_{ \pm}\left(x_{0}\right), c_{ \pm}\left(x_{0}\right)\right)$ which are optimal with respect to $\left\{\eta_{+}\right\}$and $\left\{\eta_{-}\right\}$, respectively, then, in a generic sense, the Skiba set with respect to $\mathcal{Y}=\left\{\eta_{+}, \eta_{-}\right\}$is locally a manifold of dimension $n-1$. In particular it is a point if there is one state variable and it is a curve if there are two.

This can be seen by the following arguments:

Let $\hat{U}_{ \pm}\left(x_{0}\right)$ be the value of the objective function at $\left(x_{ \pm}\left(x_{0}\right), c_{ \pm}\left(x_{0}\right)\right)$ for each $x_{0} \in V$, then we require $\hat{U}_{+}\left(\bar{x}_{0}\right)=\hat{U}_{-}\left(\bar{x}_{0}\right)$ for a Skiba point $\bar{x}_{0}$.

The sets $W_{ \pm}=\left\{\left(x_{0}, \hat{U}_{+}\left(x_{0}\right)\right) \mid x_{0} \in \mathbb{R}^{n}\right\}$ are manifolds of dimension $n$ in $\mathbb{R}^{n+1}$. Moreover, generically they intersect transversally at $\left(\bar{x}_{0}, \hat{U}_{+}\left(\bar{x}_{0}\right)\right)$, hence $T_{\bar{x}_{0}} W_{+}+$ $T_{\bar{x}_{0}} W_{-}=\mathbb{R}^{n+1}$. Thus $T_{\bar{x}_{0}} W_{+} \cap T_{\bar{x}_{0}} W_{-}$is of dimension $2 n-(n+1)=n-1$.

REMARKS 1. The above arguments may fail if $\mathcal{Y}=\left\{y_{1}, \ldots, y_{l}\right\}$ holds with $l>2$. In this case we observe

$$
\mathcal{S}(\mathcal{Y}) \subset \bigcup_{1 \leq i<j \leq l} \mathcal{S}\left(\left\{y_{i}, y_{j}\right\}\right)
$$

Consider, for example $l>2$ in case $n=2$ where the Skiba sets $\mathcal{S}\left(\left\{y_{i}, y_{j}\right\}\right)$ form curves. If these intersect then $\mathcal{S}(\mathcal{Y})$ is not a manifold near this point.

2. If the dimension of the stable manifold of an $\eta$ is less than $n$, we have to free additional state variables by replacing further $x_{+_{i}}(0)=x_{0_{i}}, x_{-_{i}}(0)=x_{0_{i}}$ by $x_{+i}(0)=x_{-i}(0)$. In this case the assumptions of Conclusion 11 are not satisfied and hence the arguments above fail.

3. If the dimension of the stable manifold of an $\eta$ is large than $n$, we may fix additional costate variables and apply the method above. A problem is, that this typically will not be optimal with respect to $\{\eta\}$ and it is not clear whether parameter continuation of the fixed costate variables really terminates in an optimum.

\section{Optimal investment}

We analyze a model of optimal investment and consumption policy which is presented in [29]. There it is shown that a non-explosiveness condition yields a region in state space, where the borrower remains credit-worthy.

The full problem can be split into the problem of optimal investment for maximizing the wealth of the economy and into the problem of optimal consumption policy to maximize the utility of this wealth.

We restrict to the problem of optimal investment. Using Pontryagin's maximum principle yields a 2-dimensional dynamical system in state-costate space. It will 
be seen that the stable manifolds of stationary points are candidates for optimal solutions.

\section{The model}

There are two control variables, the investment $j(t)$ and the consumption $c(t) \geq 0$. The two state equations for the per capita capital stock $k(t) \geq 0$ and the per capita stock of debt $b(t)$ are:

$$
\begin{aligned}
\dot{k} & =i(k, j), & k(0) & =k_{0} \\
\dot{b} & =\theta b+c-f(k, j), & b(0) & =b_{0}
\end{aligned}
$$

with the net investment function $i(k, j)=j-\sigma k$ (depreciation rate $\sigma$ ), net income function $f(k, j)=c_{f} k^{\alpha}-\left(j+\frac{j^{2}}{k^{\gamma}}\right), k>0, f(0,0)=0$ and the rate of interest $\theta$.

We assume $\sigma \geq 0, \theta \geq 0, c_{f} \geq 0, \gamma \in(0,1), 0<\alpha<2-\gamma$.

Moreover, the following non-explosiveness condition for the debt is required:

$$
\lim _{t \rightarrow \infty} e^{-\theta t} b(t)=0
$$

This asymptotic boundary condition implies that the state variables have to stay bounded if the borrower remains credit-worthy (see [29]).

To optimize the utility $\hat{U}(c):=\int_{0}^{\infty} e^{-\theta t} U(c(t)) \mathrm{dt}$ we have to solve

$$
\begin{cases}\max _{c, j} \int_{0}^{\infty} e^{-\theta t} U(c(t)) \mathrm{dt}, & \\ \dot{k}=i(k, j) ; & (0)=k_{0}, \\ \dot{b}=\theta b+c-f(k, j) ; & b(0)=b_{0}, \\ \lim _{t \rightarrow \infty} e^{-\theta t} b(t)=0, & \end{cases}
$$

where $U$ is a strictly monotone increasing utility function.

This problem can be separated into two optimization problems.

a) Solve the investment problem for $k_{0} \in \mathbb{R}_{+}$

$$
\left\{\begin{array}{l}
\max _{j} \int_{0}^{\infty} e^{-\theta s} f(k(s), j(s)) \mathrm{d} s, \quad k(0)=k_{0} . \\
\dot{k}=i(k, j)
\end{array}\right.
$$

By using an optimal solution $\left(k^{*}(t), j^{*}(t)\right)$ of $\left(P_{I}\left(k_{0}\right)\right)$ we define the wealth of the economy at time $t=0$ by $w^{*}:=\int_{0}^{\infty} e^{-\theta s} f\left(k^{*}(s), j^{*}(s)\right) \mathrm{ds}-b_{0}$.

b) Solve the problem of optimal consumption for given $\left(k, j, b_{0}\right)$, and $w \in \mathbb{R}_{+}$

$$
\left\{\begin{array}{l}
\max _{c, \bar{c} \leq w} \int_{0}^{\infty} U(c(s)) e^{-\theta s} \mathrm{ds}, \\
\dot{b}=\theta b+c-f(k, j), \\
\lim _{t \rightarrow \infty} e^{-\theta t} b(t)=0,
\end{array} \quad b(0)=b_{0}, \quad\left(P_{C}\left(w, k, j, b_{0}\right)\right)\right.
$$

where $\bar{c}:=\int_{0}^{\infty} e^{-\theta s} c(s) \mathrm{ds}$. We denote a solution of $\left(P_{C}\left(w, k, j, b_{0}\right)\right)$ by $\left(b^{*}(t), c^{*}(t)\right)$. 
It can be seen, that $(\tilde{k}, \tilde{b}, \tilde{j}, \tilde{c})$ is an optimal solution of $\left(P_{F}\left(k_{0}, b_{0}\right)\right)$ if and only if $(\tilde{k}, \tilde{j})$ is an optimal solution of $\left(P_{I}\left(k_{0}\right)\right)$ and $(\tilde{b}, \tilde{c})$ is an optimal solution of $\left(P_{C}\left(\tilde{w}, \tilde{k}, \tilde{j}, b_{0}\right)\right)$, where $\tilde{w}:=\int_{0}^{\infty} e^{-\theta s} f(\tilde{k}(s), \tilde{j}(s)) \mathrm{ds}-b_{0}$.

An analytical treatment why and under what conditions such problems can be separated is given in [31]. Henceforward we treat $\left(P_{I}\left(k_{0}\right)\right)$. Let us apply Theorem 5 to this system.

Theorem 12 A necessary condition for $\left(k^{*}(t), j^{*}(t)\right), 0 \leq t<\infty$, to be an optimal solution of $\left(P_{I}\left(k_{0}\right)\right)$ is that there exist a real number $\lambda \in\{0,1\}$ and an initial costate value $x_{0} \in \mathbb{R}$, such that

1. $\left(\lambda, x_{0}\right) \neq 0$.

2. The costate variable $x(\cdot)$ is the continuous and piecewise differentiable solution of

$$
\left\{\begin{array}{l}
\dot{x}(t)=-\lambda e^{-\theta t} \frac{\partial}{\partial k} f\left(k^{*}(t), j^{*}(t)\right)-x(t) \frac{\partial}{\partial k} i\left(k^{*}(t), j^{*}(t)\right) \\
x(0)=x_{0}
\end{array}\right.
$$

3. The investment $j^{*}(t)$ maximizes the Hamiltonian function

$$
H\left(k^{*}(t), j, x(t), \lambda, t\right)=\lambda e^{-\theta t} f\left(k^{*}(t), j\right)+x(t) i\left(k^{*}(t), j\right),
$$

at each $t$ where $j^{*}(t)$ is continuous.

4. The maximum of the Hamiltonian satisfies for every $t$

$$
M(t):=H\left(k^{*}(t), j^{*}(t), x(t), \lambda, t\right)=-q(t),
$$

where the function $q(t)$ is the solution of

$$
\left\{\begin{array}{l}
\dot{q}=\theta e^{-\theta t} f\left(k^{*}(t), j^{*}(t)\right) \\
\lim _{t \rightarrow \infty} q(t)=0
\end{array}\right.
$$

Now we consider the special choice for $f, i$ above and draw some conclusions from this theorem. Let $\left(k^{*}, j^{*}\right)$ be an optimal solution of $\left(P_{I}\left(k_{0}\right)\right)$ and let $\lambda, x_{0}, x$ and $q$ be as in Theorem 12 .

- If $\lambda=0$, then 1., 2. imply $x_{0} \neq 0$ and $\dot{x}(t)=x(t) \sigma$ (notice $i(k, j)=j-\sigma k$ ), hence $x(t)=e^{\sigma t} x_{0} \neq 0$. Thus $\frac{\partial}{\partial j} H\left(k^{*}(t), j, x(t), a, t\right)=x(t) \frac{\partial}{\partial j} i\left(k^{*}(t), j\right)=$ $x(t) \neq 0$. This contradicts condition 3. and hence $\lambda=1$.

- Condition 3. implies $j^{*}(t)=j\left(k^{*}(t), e^{\theta t} x(t)\right)$ for each $0 \leq t<\infty$, where $j(k, y)=\frac{y-1}{2} k^{\gamma}$. Continuity of $x$ and $k^{*}$ imply continuity of $j^{*}$. Thus, an optimal solution must have a continuous control. 
- The scaled function

$$
(k(t), y(t)):=\left(k^{*}(t), e^{\theta t} x(t)\right)
$$

is a solution of

$$
\left(\begin{array}{c}
\dot{k} \\
\dot{y}
\end{array}\right)=\left(\begin{array}{c}
\frac{y-1}{2} k^{\gamma}-\sigma k \\
(\theta+\sigma) y-\alpha c_{f} k^{\alpha-1}-\gamma \frac{(y-1)^{2}}{4} k^{\gamma-1}
\end{array}\right)=: F(k, y)
$$

with $k(0)=k_{0}, y(0)=y_{0}:=x_{0}$.

- By integration (28) implies $q(t)=-\theta \int_{t}^{\infty} e^{-\theta s} f\left(k^{*}(s), j^{*}(s)\right) \mathrm{d} s$.

- The definition of $y$ and 4. yields for each $t$ :

$$
y(t) \dot{k}^{*}(t)=\theta \int_{t}^{\infty} e^{-\theta(s-t)} f\left(k^{*}(s), j^{*}(s)\right) \mathrm{ds}-f\left(k^{*}(t), j^{*}(t)\right) .
$$

For the phase portrait of the dynamical system (29) see Figure 1.

The system has two stationary points, $\left(k_{u}, y_{u}\right)$ is a sink and $\left(k_{s}, y_{s}\right)$ is a hyperbolic saddle.

We approximate a solution $z_{+}(t)=\left(k_{+}(t), y_{+}(t)\right)$ on the stable manifold of $\left(k_{s}, y_{s}\right)$ by the techniques from Section 2 by solving the boundary value problem

$$
\begin{aligned}
\left(\begin{array}{c}
\dot{k}(t) \\
\dot{y}(t)
\end{array}\right) & =F(k(t), y(t)), t \in\left[0, T_{+}\right], \\
V\left(\left(\begin{array}{l}
k\left(T_{+}\right) \\
y\left(T_{+}\right)
\end{array}\right)-\left(\begin{array}{c}
k_{s} \\
y_{s}
\end{array}\right)\right) & =0, \\
k(0) & =k_{0},
\end{aligned}
$$

where $V^{T}$ is the eigenvector of $F^{\prime}\left(k_{s}, y_{s}\right)^{T}$ corresponding to the unstable eigenvalue. The solution $z_{+}(t)=\left(k_{+}(t), y_{+}(t)\right)$ is drawn in Figure 1. Moreover, we define the value of the objective function by $\hat{F}_{+}\left(k_{0}\right):=\int_{0}^{\infty} e^{-\theta s} f\left[k_{+}(s), j\left(z_{+}(s)\right)\right] \mathrm{ds}$. These values are plotted in Figure 2 as a function of $k_{0}$ (with sign + ).

The only solution of (29) which 'converges' to $\left(k_{u}, y_{u}\right)$ is the stationary solution itself and its value of the objective function $\frac{f\left(k_{u}, y_{u}\right)}{\theta}$ is not optimal (see Figure 3).

For $k_{0}$ smaller than some critical value $k_{2}$ (see Figure 1 ) there is another trajectory $z_{-}(t):=\left(k_{-}(t), y_{-}(t)\right)$ of $(29)$ which converges to the singular point $(0,1)$. In fact this trajectory reaches $(0,1)$ in finite time $\hat{T}$ and it defines another bounded admissible solution of $\left(P_{I}\left(k_{0}\right)\right)$ by

$$
(k(t), j(t))=\left\{\begin{array}{cc}
\left(k_{-}(t), j\left(k_{-}(t), y_{-}(t)\right)\right) & \text { for } \quad t \in[0, \hat{T}), \\
(0,0) & \text { for } \quad t \in[\hat{T}, \infty) .
\end{array}\right.
$$

The value of its objective function is $\hat{F}_{-}\left(k_{0}\right):=\int_{0}^{\infty} e^{-\theta s} f\left[k_{-}(s), j\left(z_{-}(s)\right)\right] \mathrm{ds}$. These values are plotted in Figure 2 as a function of $k_{0}($ with sign $\times)$. 


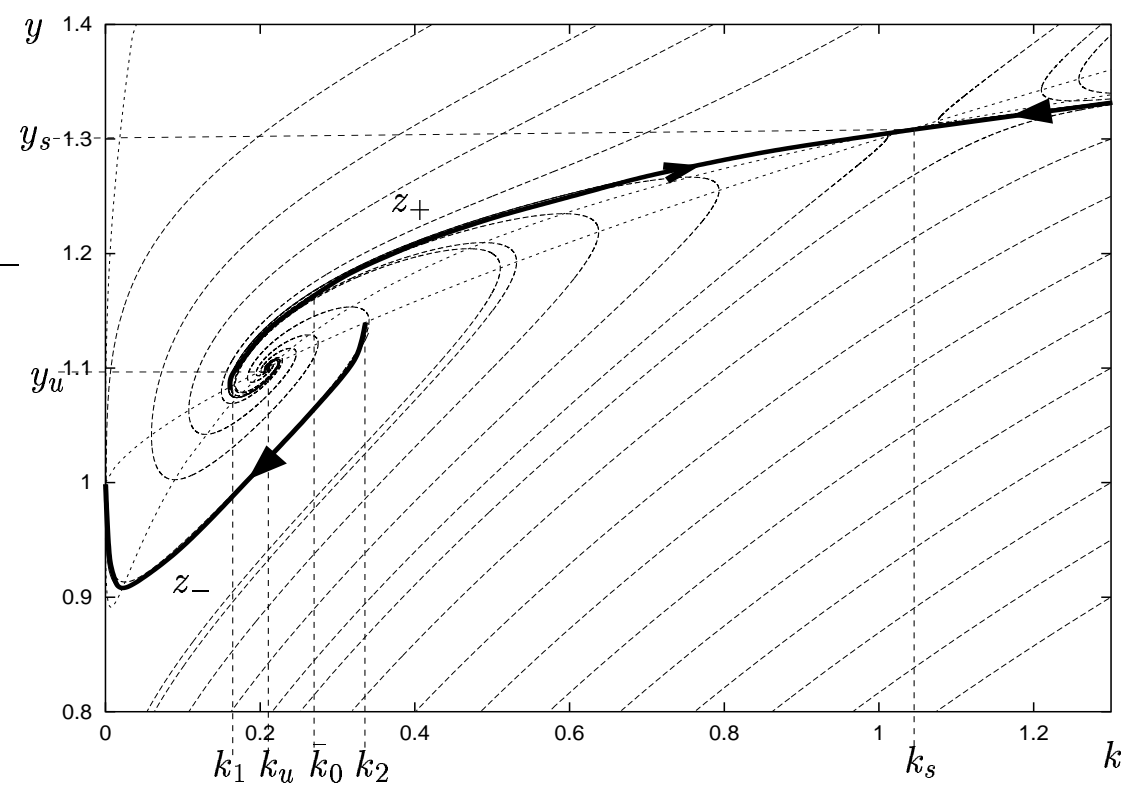

Figure 1: $k$ - $y$ phase portrait for (29) with parameters $\alpha=1.1, c_{f}=0.29, \sigma=0.15$, $\theta=0.1$ and $\gamma=0.3$.

Since the point $(0,1)$ is singular and at the boundary of the state space, we cannot apply our approximation method from Section 2. The solution, however, is very interesting because it describes a strategy where the complete capital stock is spent in finite time. In order to approximate this solution near $(0,1)$ we use an analytical approximation near singularities (compare [8]) as follows

$$
\begin{aligned}
K(t) & :=0+b_{1} t^{\epsilon_{1}}+b_{2} t^{\epsilon_{2}} \\
Y(t) & :=1+a_{1} t^{\delta_{1}}+a_{2} t^{\delta_{2}} .
\end{aligned}
$$

The coefficients $a_{1}, a_{2}, b_{1}, b_{2}, 0<\delta_{1}<\delta_{2}$ and $0<\epsilon_{1}<\epsilon_{2}$ are chosen, such that $(K(t), Y(t))$ satisfies (for suitable $\delta_{3}>0$ and $\epsilon_{3}>0$ )

$$
\left(\begin{array}{c}
\dot{K}(t) \\
\dot{Y}(t)
\end{array}\right)+F(K(t), Y(t))=\left(\begin{array}{c}
O\left(t^{\epsilon_{3}}\right) \\
O\left(t^{\delta_{3}}\right)
\end{array}\right)
$$

where $F$ is defined by $(29)$ and $p(t)=O\left(t^{\delta}\right)$ means that $\lim _{t \rightarrow 0} \frac{p(t)}{t^{\delta}}$ exists. In this particular case with $1<\alpha<\frac{3-\gamma}{2}$, the exponents are $\delta_{1}=1, \epsilon_{1}=\frac{2}{1-\gamma}$, $\delta_{2}=\frac{2(\alpha-1)}{1-\gamma}+1$ and $\epsilon_{2}=\frac{2 \alpha}{1-\gamma}$ and the coefficients are $a_{1}=-(\sigma+\theta)(1-\gamma)$, $b_{1}=\left(\frac{(\sigma+\theta)(1-\gamma)^{2}}{4}\right)^{\frac{1}{1-\gamma}}, a_{2}=\frac{\alpha-\gamma}{\delta_{2}} c_{f} b_{1}^{\alpha-1}$ and $b_{2}=-\frac{c_{f} b_{1}^{\alpha}}{\delta_{2}(\theta+\sigma)(1-\gamma)}$. The remaining exponents are $\epsilon_{3}=\frac{4(\alpha-1)}{1-\gamma}+\frac{1+\gamma}{1-\gamma}$ and $\delta_{3}=\frac{4(\alpha-1)}{1-\gamma}$. Thus $(K, Y)$ is an approximation for a solution of the time inverted system (29) with $K(0)=0, Y(0)=1$. We 


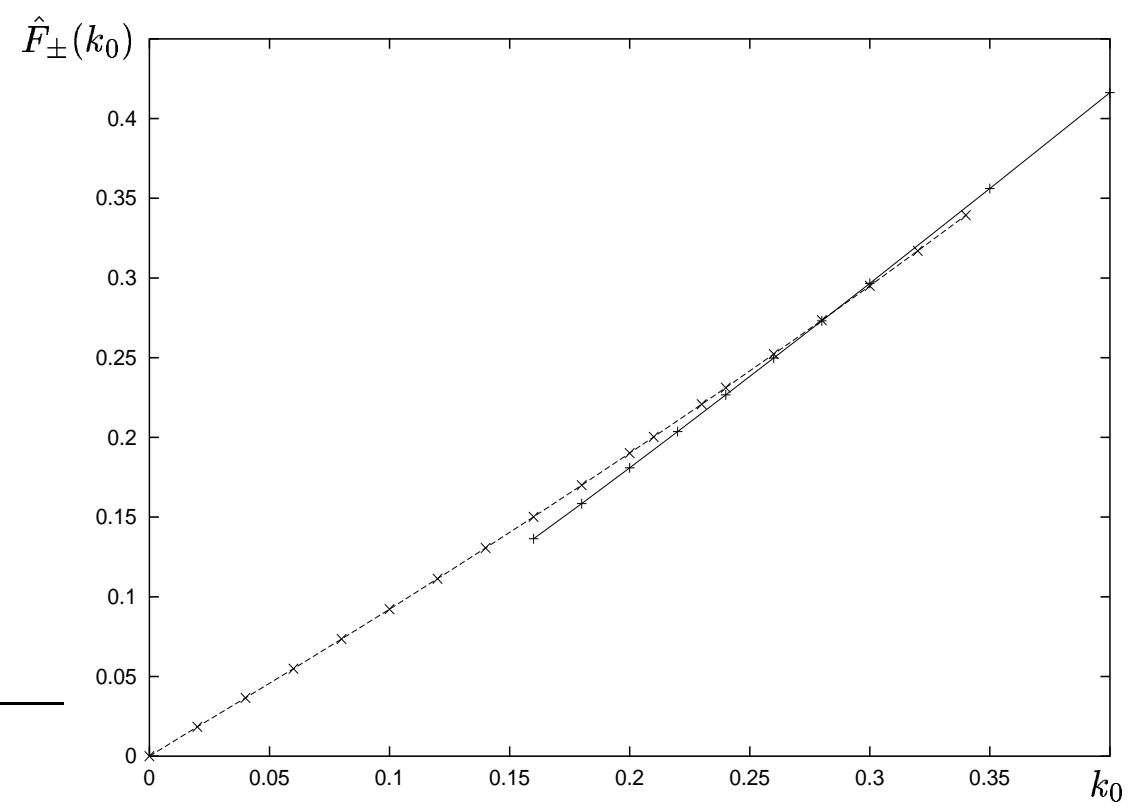

Figure 2: Values of the objective functions $\hat{F}_{-}\left(k_{0}\right)$ for $z_{-}(\times---\times)$and $\hat{F}_{+}\left(k_{0}\right)$ $(+-+)$ for $z_{+}$, depending on the initial state $k_{0}$.

choose some (small) $\bar{T}>0$, compute $\bar{K}=K(\bar{T}), \bar{Y}=Y(\bar{T})$ and solve the following boundary value problem on $[0,1]$

$$
\begin{aligned}
\left(\begin{array}{l}
\dot{k} \\
\dot{y}
\end{array}\right) & =T \cdot F(k, y), \\
\dot{T}(t) & =0 \\
k(0) & =k_{0} \\
k(1) & =\bar{K} \\
y(1) & =\bar{Y}
\end{aligned}
$$

where $T$ is an additional "free" parameter, defining the time needed to reach $(\bar{K}, \bar{Y})$. Thus we obtain an approximation for $z_{-}=\left(k_{-}, y_{-}\right)$by

$$
\left(k_{-}(t), y_{-}(t)\right)=\left\{\begin{array}{clc}
\left(k\left(\frac{t}{T}\right), y\left(\frac{t}{T}\right)\right) & : & t \in[0, T] \\
(K(\bar{T}+T-t), Y(\bar{T}+T-t)) & : & t \in(T, \bar{T}+T] \\
(0,1) & : & t>\bar{T}+T .
\end{array}\right.
$$

In particular, we obtain an approximation of the time $\hat{T}=\bar{T}+T$ which is needed to reach $k=0$.

All solutions which leave the region $\mathcal{R}:=\left\{(k, y)|| y \mid \leq \sqrt{4 c_{f} k^{\alpha-\gamma}+1}\right\}$, where $f\left(k, \frac{y-1}{2} k^{\gamma}\right) \geq 0$, and stay outside do not satisfy (30), because when they pass the boundary of $\mathcal{R}$ for the last time the right hand side of (30) is negative and the left hand side is positive. The only solutions which do not leave this region $\mathcal{R}$ are those converging to $(0,1),\left(k_{u}, y_{u}\right)$ or $\left(k_{s}, y_{s}\right)$. 
Any solution of $(29)$ with recurrence in the state variable and different value of the costate variable at the recurrence time (i. e. $k(\bar{t})=k_{0}, y(\bar{t}) \neq y(0)=x_{0}$ for some $\bar{t}>0$ ) cannot be optimal because $j(k, y)=\frac{y-1}{2} k^{\gamma}$ implies for the control $j(\bar{t}) \neq j(0)$ and Proposition 8 excludes optimality of such trajectory. This has been confirmed by numerical calculations.

Hence we can restrict to those solutions which converge monotonically to one of the steady states. Let $k_{1}$ be the minimum value of $k_{+}(t)$ and $k_{2}$ be the the maximum value of $k_{-}(t)$ (compare $z_{+}$and $z_{-}$in Figure 1 ). If $k(0) \leq k_{2}$, then the solution defined by $z_{-}$which converges to $(0,1)$ is one candidate, if $k(0) \geq k_{1}$, then the solution defined by $z_{+}$which converges to $\left(k_{s}, y_{s}\right)$ is another candidate. At $k(0)=k_{u}$ the stationary solution $\left(k_{u}, j_{u}\right), j_{u}=\sigma k_{u}$ is another candidate, but in a neighborhood of $k_{u}$ there exists no solution converging to $k_{u}$ and hence $k_{u}$ is not nondegenerate. Moreover, the value of its objective function is not optimal (see Figure 3).

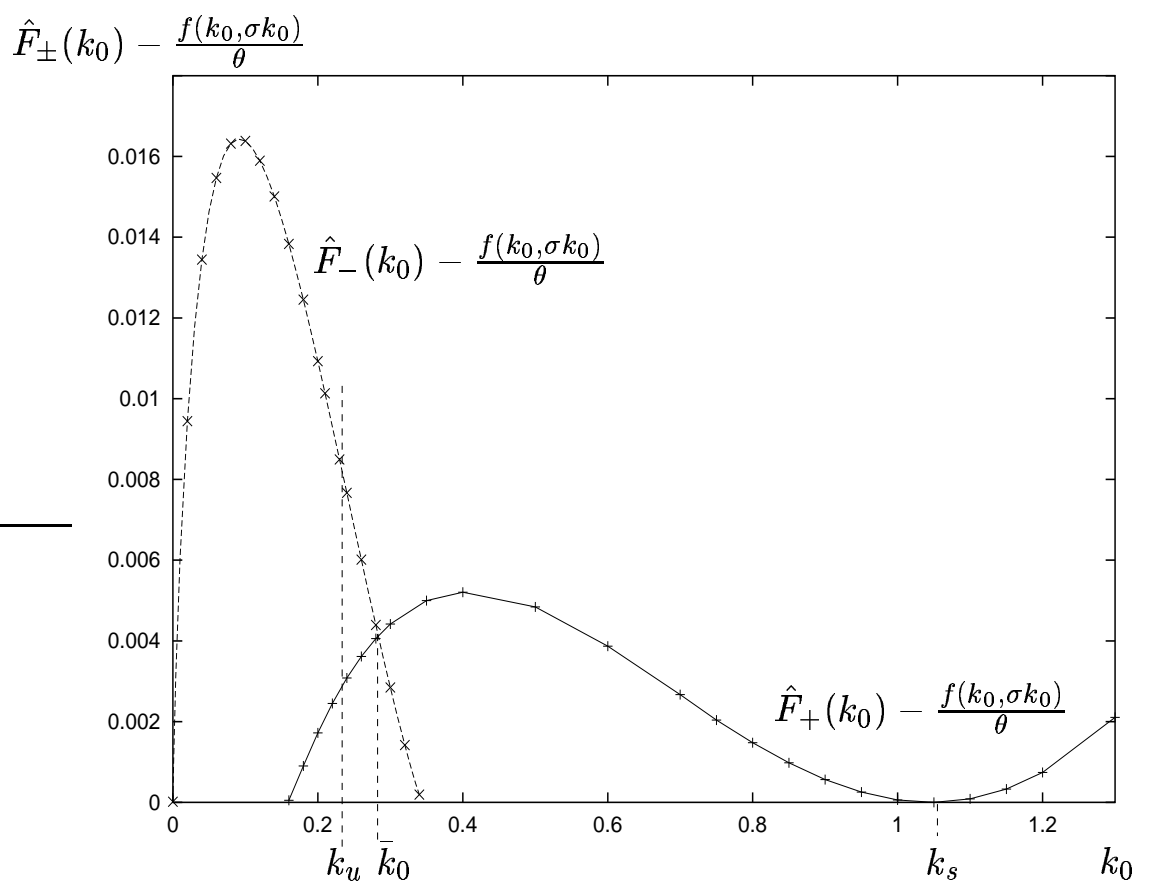

Figure 3: Difference between the objective values $\hat{F}_{ \pm}\left(k_{0}\right)$ of $z_{ \pm}$and the objective value $\frac{f\left(k_{0}, \sigma k_{0}\right)}{\theta}$ of the stationary control $j(t):=\sigma k_{0}$ for different $k_{0}$.

If we compute the values of the objective function we get Figure 2. In Figure 3 we show the difference of the values of the objective function to $\frac{f\left(k_{0}, \sigma k_{0}\right)}{\theta}$ which is the value of the objective function of the admissible stationary solution of $\left(P_{I}\left(k_{0}\right)\right)$ defined by $\left(k_{0}, \sigma k_{0}\right)$ (notice that this solution does not solve the differential equation (29)).

Figure 3 shows that there is a Skiba point at a value $\bar{k}_{0} \approx 0.28$. Notice that this is not $k_{u}$, the value of the unstable steady state. 
At the Skiba point the global optimal strategy changes, for an initial capital stock less than $\bar{k}_{0}$ the optimal strategy is to "sell" the capital stock and for an initial capital stock larger than $\bar{k}_{0}$ it is optimal to reach the upper steady state.

\section{Resource management}

In [25] a model of optimization in resource management is presented and the solutions near a steady state are analyzed. The existence of periodic orbits for rather large discount rates is proved in [13] by detecting a Hopf bifurcation.

We analyze a similar model of optimal exploitation of renewable resources and optimal consumption policy due to [30].

We demonstrate in this section the existence of periodic orbits for the latter system and show how to approximate solutions converging to it by using the method from $[22]$.

Using Pontryagin's maximum principle we get a 4-dimensional ordinary differential equation and we detect periodic orbits by parameter continuation and bifurcation theory using CONTENT [17].

\section{The model}

The control variables are the rate of extraction of resources $0 \leq q(t) \leq \bar{Q}$ and the consumption policy $0 \leq c(t) \leq \bar{C}$. The change of the stock of resource $R(t)$ and the level of countries debt $B(t)$ is given by

$$
\begin{array}{rlrl}
\dot{R} & =g(R)-q R, & & R(0)=R_{0}, \\
\dot{B} & =h(B)-f(q R)+c, & B(0)=B_{0}
\end{array}
$$

where $g(R)=e R(1-R), e>0$ is the natural growth function of resource and the costs of debt are $h(B)=a B^{\sigma}, a>0, \sigma>1$. The production function is defined by $f(D)=p\left((1+D)^{\gamma}-1\right), 0<\gamma<1$, where $D$ is the amount of used resources.

There exists a critical curve $x^{*}(R)$, such that it is possible to hold debt bounded if $B(t) \leq x^{*}(R(t))$. In [30] it is shown how to compute this curve by using vector field analysis.

We want to optimize the utility stream over an infinite time horizon

$$
\int_{0}^{\infty} U(c(t), R(t)) e^{-\delta t} \mathrm{dt}
$$

with a discount rate $\delta>0$ and a utility function $U(c, R)=d c^{\epsilon} R^{\rho}, 0<\epsilon, \rho<1$.

Using Pontryagin's maximum principle (Theorem 5) and scaling the costate variables with $e^{\delta t}$ we get necessary condition for an optimal solution. In particular, it turns out that only $\lambda=1$ is possible. Defining scaled costate variables $x, y$ we obtain, that an optimal solution $(R, B, x, y)$ solves the 4-dimensional dynamical system 


$$
\begin{aligned}
\dot{R} & =e R(1-R)-q R ; & R(0)=R_{0} \\
\dot{B} & =a B^{\sigma}-p\left((1+q R)^{\gamma}-1\right)+c ; & B(0)=B_{0} \\
\dot{x} & =x \delta-x e(1-2 R)-d \rho c^{\epsilon} R^{\rho-1} & \\
\dot{y} & =y\left(\delta-a \sigma B^{\sigma-1}\right) &
\end{aligned}
$$

with $q=\left(\left(-\frac{y p \gamma}{x}\right)^{\frac{1}{1-\gamma}}-1\right) / R$ and $c=\left(-\frac{d R^{\rho} \epsilon}{y}\right)^{\frac{1}{1-\epsilon}}$.

As in [30] we start with parameters $\gamma=0.76, \sigma=1.01, d=0.19, \epsilon=0.21, a=$ $0.1, e=0.08, \rho=0.77, \delta=0.1, p=24.85$ and we detect two steady states

$$
\left(R_{E 1}, B_{E 1}, x_{E 1}, y_{E 1}\right)=(0.5762,0.3697,1.1738,-0.0624)
$$

which leads to $\left(c_{E 1}, q_{E 1}\right)=(0.3315,0.0339)$ and

$$
\left(R_{E 2}, B_{E 2}, x_{E 2}, y_{E 2}\right)=(0.0272,0.3697,4.174,,-0.2211)
$$

which leads to $\left(c_{E 2}, q_{E 2}\right)=(0.0034,0.0778)$.

For further analysis of the system we use the continuation software CONTENT to detect Hopf bifurcations and hence periodic orbits in this system. CONTENT has been developed by Kuznetsov and V. V. Levitin (see [17]).

We follow the branch of steady states by variation of the parameter $\delta$ and reach a limit point. By continuation of the limit point in the 2-dimensional $(\epsilon, \delta)$-parameter space we reach a Takens-Bogdanov point at $\epsilon_{T B}=0.52737, \delta_{T B}=0.10107$.

At a Takens-Bogdanov bifurcation a branch of homoclinic orbits and a branch of Hopf points emanate (see [16]). For details on the numerical treatment of a TakensBogdanov bifurcation and the continuation of the branch of homoclinic orbits see [6].

We follow the branch of Hopf points from $\left(\epsilon_{T B}, \delta_{T B}\right)$ to $(\epsilon, \delta)=(0.6118,0.1)$.

At the branch of Hopf bifurcations unstable periodic orbits arise (no stable direction).

We follow the branch of periodic orbits by continuation of the parameter $\epsilon$ and for constant $\delta=0.1$. This yields Figure 4 , where we draw $\epsilon$ versus the period of the orbit.

Starting at the Hopf point $H$ with unstable periodic orbits we reach a period doubling $P$, such that one stable direction for the periodic orbit arises. At the turning point for periodic orbits $L$ there is another period doubling and the periodic orbits which turn back have again one stable direction. Therefore, at $\epsilon=0.6075$ there are two periodic orbits for the same parameter value plotted by lines $(-)$ $($ period $T=46.9)$ and $(--)($ period $T=39.7)$ in Figure 5.

By numerical integration one observes that (24) holds and hence both periodic orbits are candidates if $\left(R_{0}, B_{0}\right)$ is on the periodic orbit. The value of the objective 


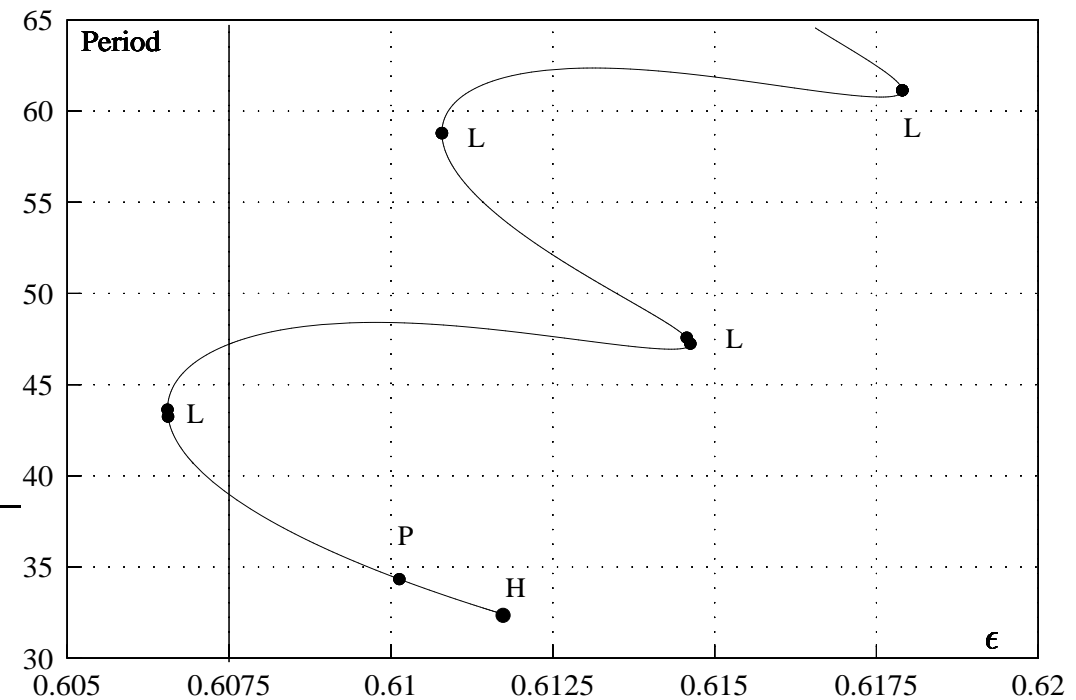

Figure 4: Continuation of periodic orbits.

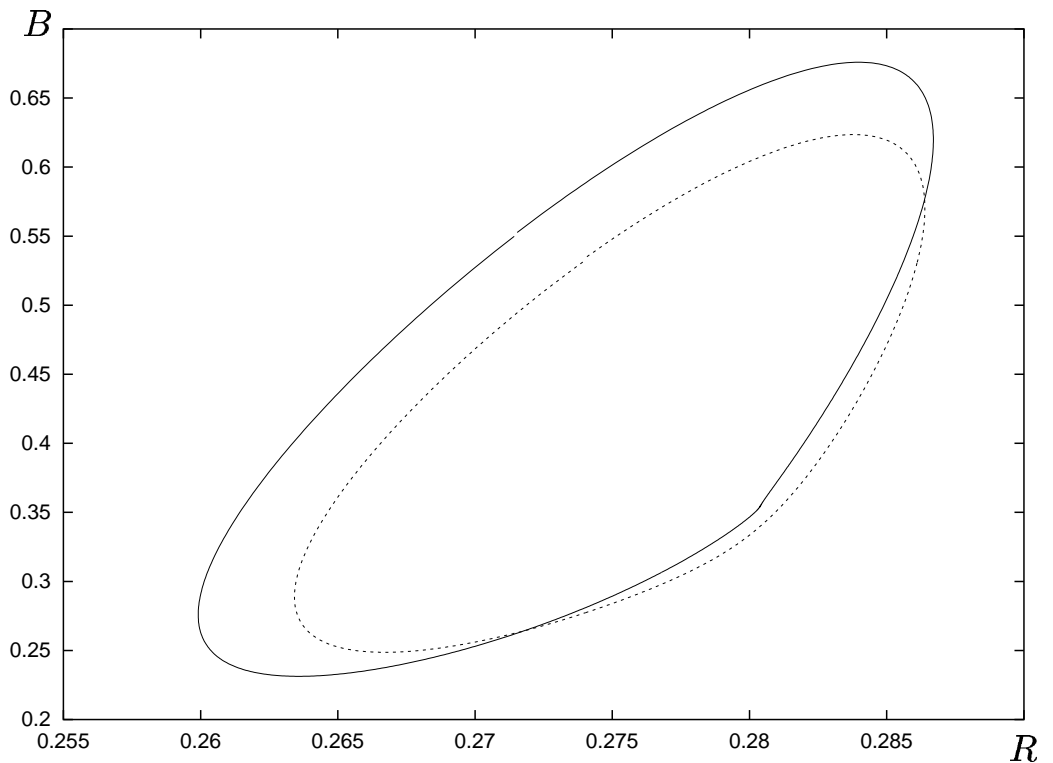

Figure 5: Projection of both periodic orbits onto the $R-B$ state space. 
function depends on the starting point $\left(R_{0}, B_{0}, x_{0}, y_{0}\right)$ on the periodic orbits and is defined by the function $v$ in (23).

In Figure 6 we plot $v$ for initial points on each of the periodic orbits in the $R-B$ space. In Figure 7 and Figure 8 we plot its projection onto the $(R, v)$ and $(B, v)$ plane, respectively.

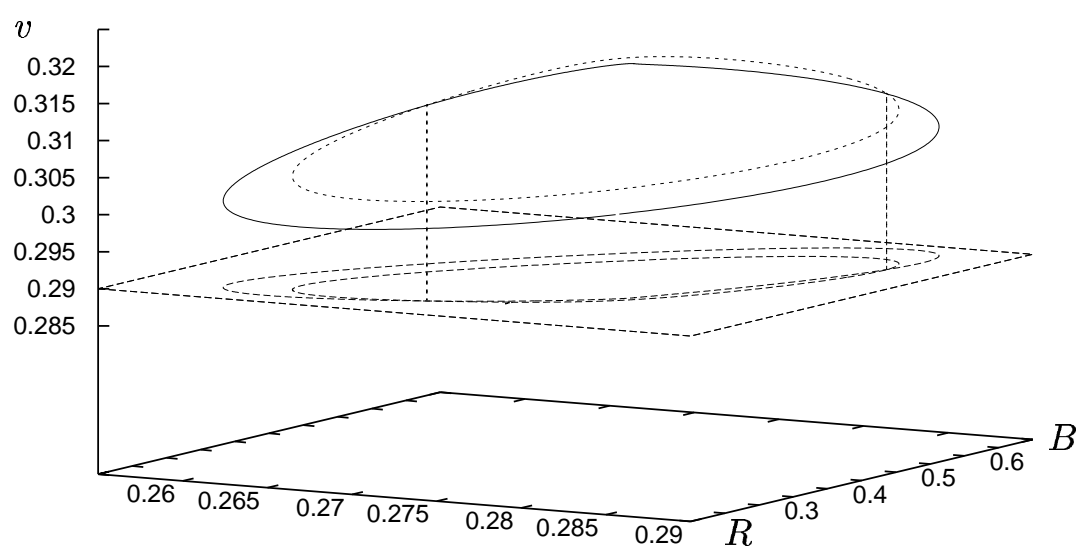

Figure 6: Values of the objective function of different initial points on both periodic orbits in the $R-B$ state space.

The numerical observations suggest that the objective functions have equal values at the periodic orbits started at their point of intersection in state space.

CONCLUSION 13 If the projection onto the state space of two periodic orbits intersect and the values of the objective functions at the intersection are equal, then none of the periodic orbits is optimal.

This can be seen by the following arguments:

If both solutions are optimal, then a solution starting on one periodic orbit and switching at the intersection to the other periodic orbit is admissible and has the same value of the objective function and hence is optimal. Thus, this solution is optimal, but its control variable is not continuous. On the other hand continuity of state and costate variables and $q=\left(\left(-\frac{y p \gamma}{x}\right)^{\frac{1}{1-\gamma}}-1\right) / R$ and $c=\left(-\frac{d R^{\rho} \epsilon}{y}\right)^{\frac{1}{1-\epsilon}}$ for optimal solutions imply continuity of the control variables. 


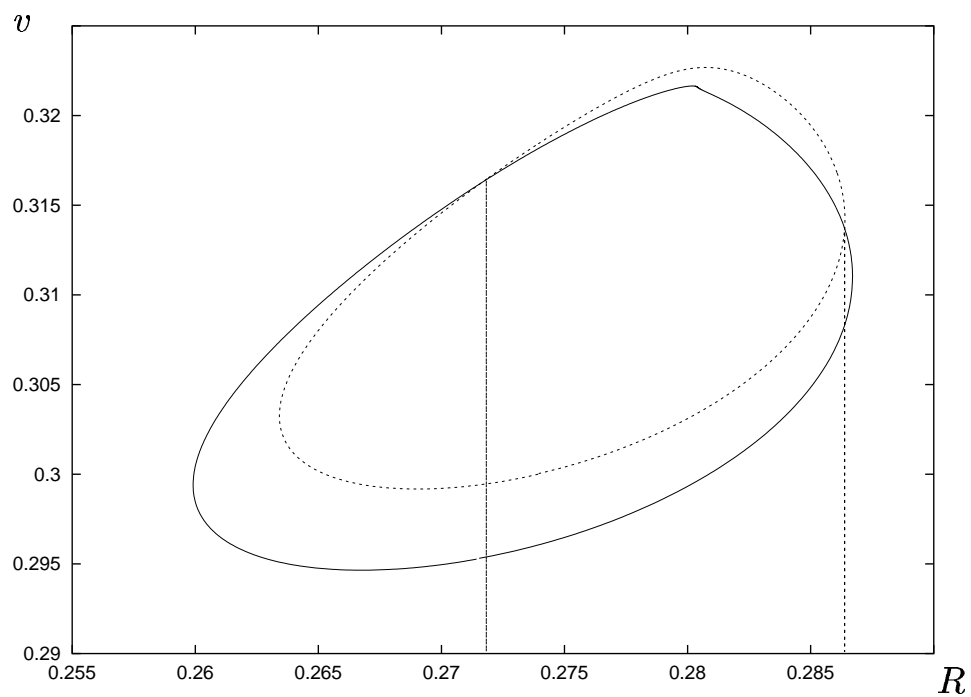

Figure 7: Values of the objective function for each of the periodic orbits parametrized by the initial values of $R$.

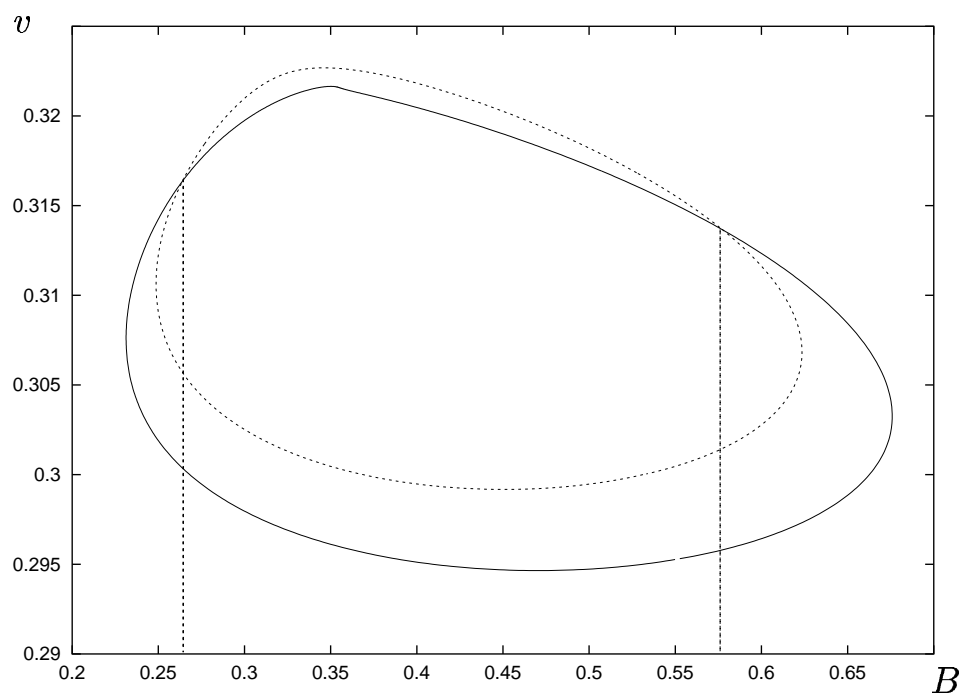

Figure 8: Values of the objective function for each of the periodic orbits parametrized by the initial values of $B$. 


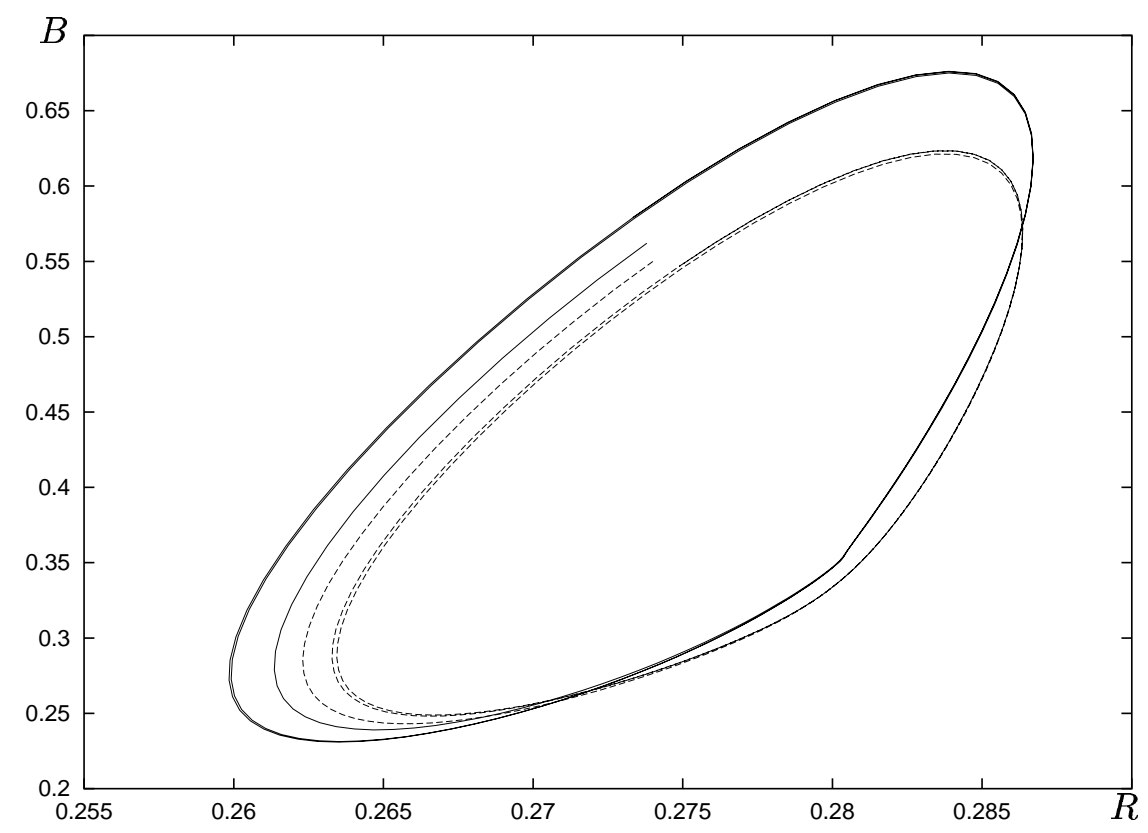

Figure 9: Projection of two approximations of solutions converging to different periodic orbits onto the $R-B$ state space.

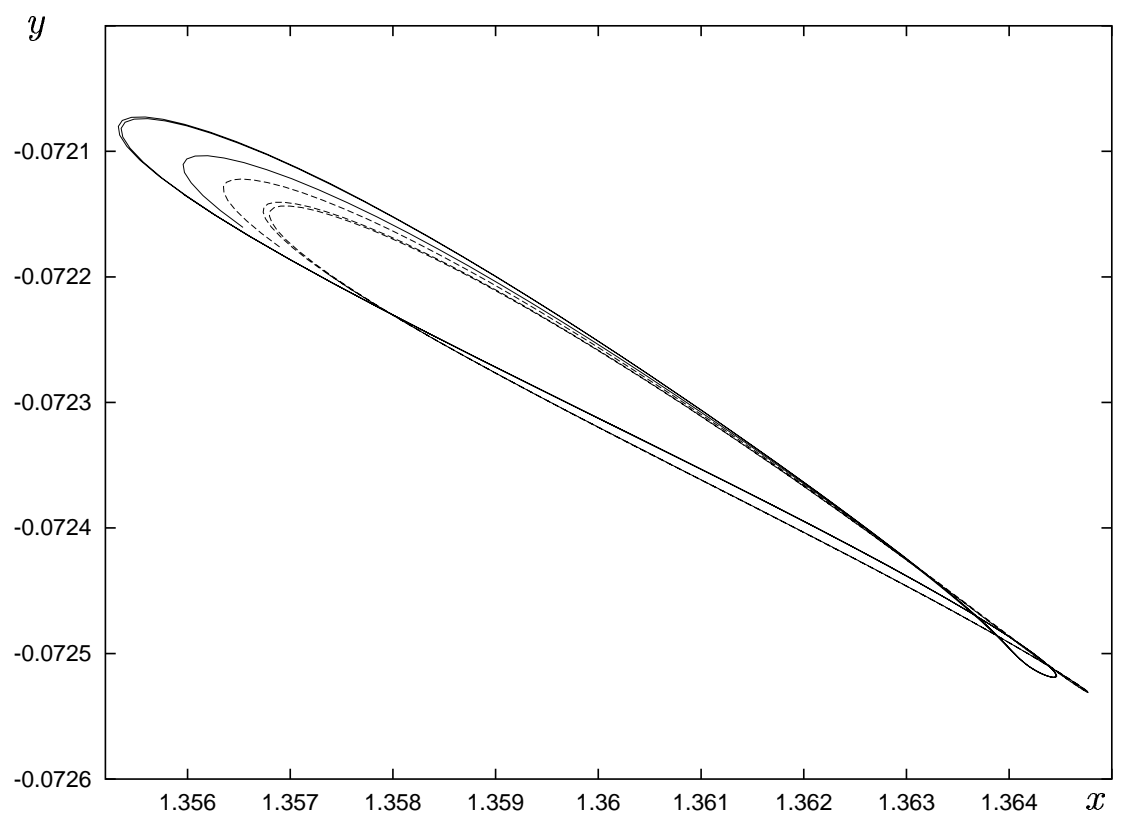

Figure 10: Projection of two approximations of solutions converging to different periodic orbits onto the $x-y$ costate space. 


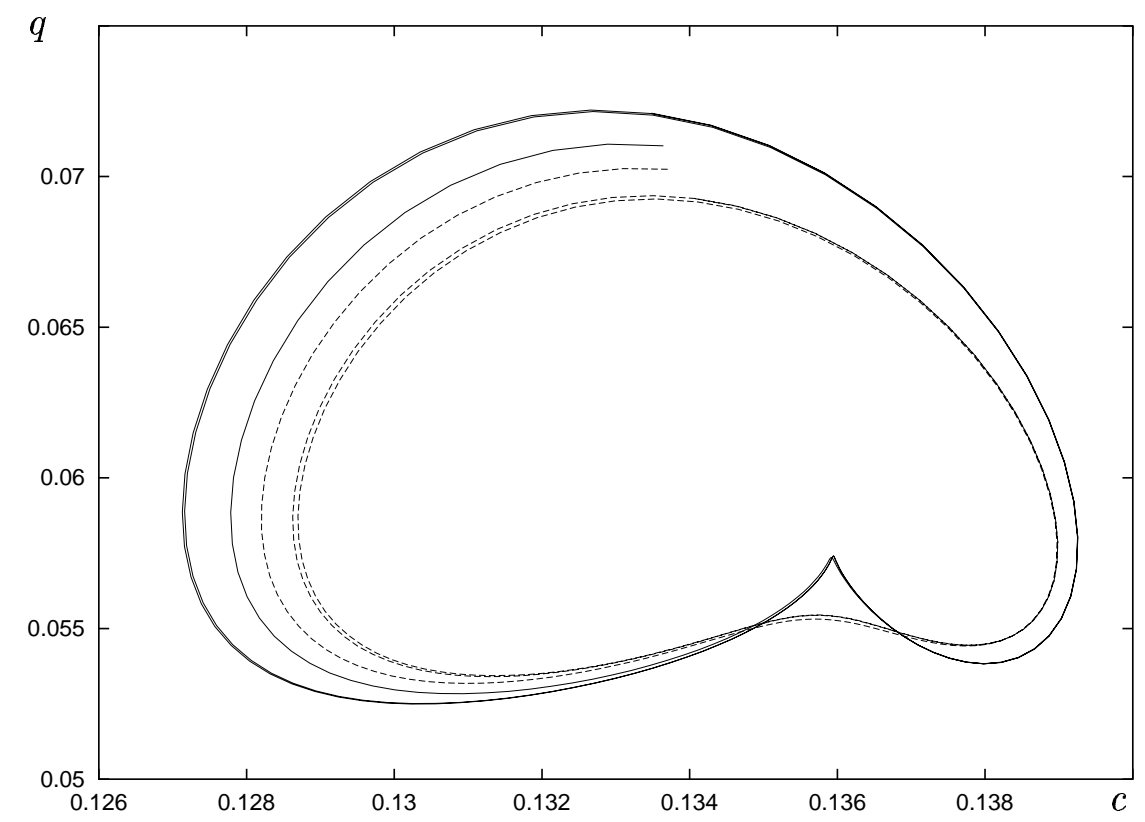

Figure 11: Control variables corresponding to the solutions converging to different periodic orbits.

Nevertheless, we approximate two solutions which converges to the periodic orbits by the boundary corrector method (12). We define $F(R, B, x, y)$ by $(31)$ and by setting $q(R, x, y)=\left(\left(-\frac{y p \gamma}{x}\right)^{\frac{1}{1-\gamma}}-1\right) / R$ and $c(R, y)=\left(-\frac{d R^{\rho} \epsilon}{y}\right)^{\frac{1}{1-\epsilon}}$. As phase condition we choose $\chi(R, B, x, y ; \varphi)=\sin (2 \pi(\varphi+\tilde{\varphi}))\left(R-R^{*}\right)-\cos (2 \pi(\varphi+\tilde{\varphi}))\left(B-B^{*}\right)$, where $\left(R^{*}, B^{*}\right)=(0.274,0.4)$ is in the interior of the projection of the periodic orbit $\tilde{R}, \tilde{B}, \tilde{x}, \tilde{y}$. Moreover, let $\tilde{\varphi}$ solve $\sin (2 \pi \tilde{\varphi})\left(\tilde{R}(0)-R^{*}\right)-\cos (2 \pi \tilde{\varphi})\left(\tilde{B}(0)-B^{*}\right)=0$ to ensure $\chi(\tilde{R}, \tilde{B}, \tilde{x}, \tilde{y} ; 0)=0$. The phase parameter $\varphi$ defines the angle of rotation around $\left(R^{*}, B^{*}\right)$.

The projection of the phase portrait onto the $R-B$ state space is drawn in Figure 9 the projection onto the $x-y$ costate space is drawn in Figure 10 and the corresponding controls are shown in Figure 11.

The structure of the dynamics near these periodic orbits is complicated and there are several periodic orbits emanating from period doublings nearby. Thus it seems that the non-degeneracy is "weak". We were not able to detect a Skiba point in this case, i. e. initial values with equal state variables and different costate variables, such that the trajectories converge to the different periodic orbits and yield the same value for the objective function. 


\section{Conclusions}

In this paper we discuss continuous optimization problems with infinite horizon. We develop a numerical tool for approximating candidates for optimal solutions or Skiba points with stationary or periodic asymptotic behavior. Moreover, this approach allows continuation of parameters to compute parts of higher dimensional Skiba sets. The main results of an error analysis for the numerical method, as presented in [22], [24], are summarized in Section 2. We apply our method to an optimal investment model, where a Skiba point occurs and to a model of optimal exploitation of renewable resources, where periodic solutions occur, but a Skiba point could not be detected.

This rather general concept is based on first order necessary conditions, hence the question whether the approximate candidates are optimal in a global sense is not resolved by our approach.

Acknowledgements We thank two anonymous referees for helpful comments which improve an earlier version of this paper.

\section{References}

[1] C. Azariadis and A. Drazen. Threshold externalities in economic development. Quarterly Journal of Economics, 105(2):501-526, 1990.

[2] G. Bader and P. Kunkel. Continuation and collocation for parameter dependent boundary value problems. SIAM J. Sci. Stat. Comput., 10:72-88, 1989.

[3] J. Benhabib and R. Perli. Uniqueness and indeterminacy: On the dynamics of endogenous growth. Journal of Economic Theory, 63(1):113-142, 1994.

[4] J. Benhabib, R. Perli, and D. Xi. Monopolistic competition, indeterminacy, and growth. Ricerche Economiche, 48:279-298, 1994.

[5] J. Benhabib, S. Schmitt-Grohe, and M. Uribe. The perils of the Taylor rules. Journal of Economic Theory, 96(1/2):40-69, 2001.

[6] W.-J. Beyn. Numerical analysis of homoclinic orbits emanating from a TakensBogdanov point. IMA J. Numer. Anal., 14:381-410, 1994.

[7] W. A. Brock and D. Starret. Nonconvexities in ecological management problems. Mimeo, University of Wisconsin, 1999.

[8] A. Coddington and N. Levinson. Theory of ordinary differential equations. McGraw-Hill, New York, 1955.

[9] E. J. Doedel. AUTO: A program for the automatic bifurcation analysis of autonomous systems. In Congressus Numerantinum 33, pages 115-146, 1981. 
[10] E. J. Doedel, M. J. Friedman, and B. I. Kunin. Successive continuation for locating connecting orbits. Numer. Algorithms, 14:103-124, 1997.

[11] E. J. Doedel, H. B. Keller, and J. P. Kernèvez. Numerical analysis and control of bifurcation problems: I. Bifurcation in finite dimensions. International Journal of Bifurcation and Chaos, 1:493-520, 1991.

[12] G. Evans, S. Honkapohja, and P. Romer. Growth cycles. The American Economic Review, 88(3):495-516, 1998.

[13] G. Feichtinger and A. Novak. A note on the optimal use of environmental resources by an indebted country. J. Institutional and Theoretical Economics, 147:547-555, 1991.

[14] A. D. Jepson and H. B. Keller. Steady state and periodic solution paths: their bifurcations and computations. In T. Küpper, H. D. Mittelmann, and H. Weber, editors, Numerical Methods for Bifurcation Problems. ISNM, volume 70, pages 219-246. Birkhäuser, 1984.

[15] P. Krugman. History versus trade. Quarterly Journal of Economics, pages 651-667, 1991.

[16] Y. A. Kuznetsov. Elements of Applied Bifurcation Theory. Number 112 in Applied Mathematical Sciences. Springer, 1995.

[17] Y. A. Kuznetsov and V. V. Levitin. CONTENT-integrated environment for analysis of dynamical systems. CWI Amsterdam, 1994. Available via $\mathrm{ftp} / \mathrm{pub} / \mathrm{CONTENT}$.

[18] P. Michel. On the transversality condition in infinite horizon optimal problems. Econometrica, 50:975-985, 1982.

[19] D. T. Mortensen. The persistence and indeterminacy of unemployment in search equilibria. Scandinavian Jorunal of Economics, 91(2):347-370, 1989.

[20] A. Orphanides and D. Zervos. Rational addiction with learning and regret. Journal of Political Economy, 103(4):739-758, 1995.

[21] A. Orphanides and D. Zervos. Myopia and addictive behavior. Economic Journal, 108:75-91, 1998.

[22] T. Pampel. Numerical approximation of generalized connecting orbits. PhDThesis, Department of Mathematics, University of Bielefeld, 1999. Available via http://archiv.ub.uni-bielefeld.de/disshabi/mathe.htm.

[23] T. Pampel. Approximation of generalized connecting orbits with asymptotic rate. Manuscript, 2001.

[24] T. Pampel. Numerical approximation of connecting orbits with asymptotic rate. Numerische Mathematik, 2001. Forthcoming, the original publication is available on LINK at http://dx.doi.org/10.1007/s002110100302. 
[25] M. Rauscher. The optimal use of environmental resources by an indebted country. J. Institutional and Theoretical Economics, 53:31-50, 1990.

[26] M. S. Santos. Non-existence of continuous markov equilibria in competitive market economies. Mimeo, University of Minnesota, 1999.

[27] A. Seierstad and K. Sydsæter. Sufficient conditions in optimal control theory. International Economic Review, 18(2):367-391, 1977.

[28] W. Semmler and A. Greiner. Monetary policy and non-uniqueness of unemployment steady states. Technical report, University of Bielefeld, Department of Economics, 1999. Paper prepared for the CEPR conference on Expectations, Economic Theory and Economic Policy, Perugia, September 1999.

[29] W. Semmler and M. Sieveking. The use of vector field analysis for studying debt dynamics. Discussion paper 379, University of Bielefeld, Department of Economics, 1998.

[30] W. Semmler and M. Sieveking. Critical debt and debt dynamics. Journal of Economic Dynamics and Control, 24(5-7):1121-1144, 2000.

[31] M. Sieveking and W. Semmler. Optimal consumption of value. Unpublished manuscript, 1998.

[32] A. K. Skiba. Optimal growth with a convex-concave production function. Econometrica, 46:527-540, 1978.

[33] A. Takayama. Mathematical Economics. Dryden Press, Hinsdale, Illinois, 1974. 